\title{
The directivity of a compact antenna: an unforgettable figure of merit
}

\author{
Richard W. Ziolkowski ${ }^{1,2, *}$ \\ ${ }^{1}$ University of Technology Sydney (UTS), Global Big Data Technologies Centre, Ultimo, NSW 2000, Australia \\ 2 The University of Arizona, Department of Electrical and Computer Engineering, Tucson, AZ 85721, USA
}

Received: 23 February 2017 / Received in final form: 28 July 2017 / Accepted: 25 August 2017

\begin{abstract}
When an electrically small antenna is conceived, designed, simulated, and tested, the main emphasis is usually placed immediately on its impedance bandwidth and radiation efficiency. All too often it is assumed that its directivity will only be that of a Hertzian dipole and, hence, its directivity becomes a minor consideration. This is particularly true if such a compact antenna radiates in the presence of a large ground plane. Attention is typically focused on the radiator and its size, while the ground plane is forgotten. This has become a too frequent occurrence when antennas, such as patch antennas that have been augmented with metamaterial structures, are explored. In this paper, it is demonstrated that while the ground plane has little impact on the resonance frequency and impedance bandwidth of patch antennas or metamaterial-inspired three-dimensional magnetic EZ antennas, it has a huge impact on their directivity performance. Moreover, it is demonstrated that with both a metamaterialinspired two-element array and a related Huygens dipole antenna, one can achieve broadside-radiating electrically small systems that have high directivities. Several common and original designs are used to highlight these issues and to emphasize why a fundamental figure of merit such as directivity should never be overlooked.
\end{abstract}

Keywords: Directivity / Electrically small antennas / Huygens source / Metamaterial-inspired antennas / Patch antennas

\section{Introduction}

As mobile platform and the internet-of-things move into serious 5G research and development efforts, one antenna/ array performance characteristic frequently mentioned is directivity. The anticipated desire for low probability of intercept and massive multiple-input multiple-output systems is immense. Efforts to satiate these demands would greatly benefit from compact, highly directive antennas and arrays. On the other hand, most current cell phones or laptop computer antennas for communications and data transfer applications have low directivities and low front-to-back ratios (FTBRs). Connectivity generally has been more important than directivity in the past.

Furthermore, there has been little discussed in the literature directly about the directivity of electrically small antennas. Since they are electrically small, the directivity of a stand-alone compact antenna tends to be assumed to be approximately $1.76 \mathrm{~dB}$, the value for an electric Hertzian dipole. Attention is then focused on the known difficult issues of $Q$-factor, bandwidth, and radiation efficiency. Nonetheless, there are instances in which directivity has

\footnotetext{
* e-mail: Richard.Ziolkowski@uts.edu.au
}

been addressed head-on, including issues when a small antenna does in fact radiate in the presence of a ground plane [1-4]. The authors, Kildal and Best, note directly with the beginning sentence in their joint paper [3]: "Publications on fundamental limitations of small antennas normally study the relation between the antenna size and its radiation $Q$-factor, whereas very few papers consider the fundamental directivity limitations."

Because antenna engineers generally have indeed been more worried about efficiency, matching, and bandwidth with compact systems, directivity often then takes a secondary role for single radiating element devices. This has even led to some misinterpretations of results, for instance, with metamaterial-augmented patch antennas. By loading the radiating patch with metamaterial constructs, the patch size can be decreased considerably. Unfortunately, the overall size of the patch antenna is not determined solely by the radiating patch size, but rather should include the ground plane size. Because the ground plane size usually is already many times larger than the patch, it does not play a role in variations in the impedance matching or radiation efficiency. On the other hand, as will be illustrated in this article, it does significantly influence its directivity properties. 
(a)

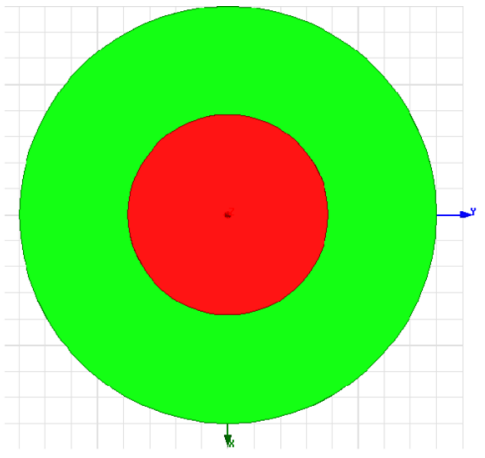

(b)

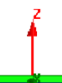

Fig. 1. Coax-fed, circular patch antenna. (a) Top view (substrate and ground plane disk have the same radius), and (b) side view (the coax feed is offset from the origin along the $-\hat{y}$ direction).

A variety of approaches to realizing higher directivity from compact antenna systems have been considered. These include, for example, end-fire arrays [5-8], electromagnetic band-gap (EBG) structures [9], high impedance surfaces and artificial magnetic conductors [10-13], nearfield resonant parasitic (NFRP) elements [14-16], nonFoster circuit-augmented antennas [17], two-element NFRP antenna arrays [18,19], and Huygens sources [2025]. The metamaterial-inspired NFRP antenna designs have led to the realization of many performance enhancements of compact systems. They will be emphasized in this article to demonstrate how interesting directivity values can be obtained with electrically small antenna systems while not forgetting other performance characteristics.

From patch antennas in Section 2, to the 3D magnetic EZ antenna in Section 3, to the use of two-element NFRP antenna arrays in Section 4, and then to a multiple NFRP element antenna in Section 5, directivity and FTBR values will be emphasized to characterize these different compact and electrically small antenna systems. In particular, these various examples will illustrate how one tailors their designs to obtain radiated power emitted primarily into one hemisphere, which would be an advantageous performance characteristic for a variety of current and future $5 \mathrm{G}$ applications. The latter include biomedical monitoring and on-body systems; point-to-point communications and wireless power transfer; mitigation of cell-phone specific absorption rate issues; and radio frequency identification devices. More power would be radiated away from the associated platforms and less power would be coupled into them. Thus, these 5G systems could evolve with lower power requirements.

All of the metal components in the reported antenna designs were taken to be copper with its known material parameters: $\varepsilon_{r}=1.0, \mu_{r}=0.999991$ and bulk conductivity $\sigma=5.8 \times 10^{7} \mathrm{~S} / \mathrm{m}$. All of the dielectric materials were taken to be the same as Rogers Duroid ${ }^{\mathrm{TM}} 5880$ with material properties: $\varepsilon_{r}=2.2, \quad \mu_{r}=1.0$ and loss tangent tan $\varphi=0.0009$. Several of the integrated components are based on the Rogers Duroid ${ }^{\mathrm{TM}} 5880$ copper cladded substrate that is $0.7874 \mathrm{~mm}$ (31 mils) thick with 0.5 oz copper, i.e., $0.017 \mathrm{~mm}$ thick copper. The operational frequencies of interest were arbitrarily selected to be near $300 \mathrm{MHz}$. The presented designs can be rescaled and tuned straightforwardly to other choices. All of the antennas are fed with a coax cable. The associated input impedance calculations were obtained by de-embedding all parameters from the port to the antenna end of the coax feed. The numerical simulations and their optimizations were carried out using the ANSYS high frequency structure simulator: ANSYS High Frequency Structure Simulator (HFSS) v15 (64-bit) [26]. While only simulated performance characteristics are considered in this article, prototypes of variations of all of the presented antennas have been successfully fabricated and tested, confirming their properties.

\section{Patch antenna}

The basic reference case is taken to be a coax-fed circular patch antenna designed to radiate close to $300 \mathrm{MHz}$. Since its introduction [27], the patch antenna has become a ubiquitous low profile antenna that has been found useful for many applications. This choice facilitates comparisons with the metamaterial-inspired designs considered in the past [14] and in this article.

The original design theory [28-30] now has a more refined result for the resonance frequency of the fundamental $\mathrm{TM}_{110}$ mode [31], Eqs. (14)-(65)]

$$
f_{\text {res,patch }}=\frac{c}{2 \pi \sqrt{\varepsilon_{r}}}\left(\frac{1.8412}{a_{\text {eff }}}\right),
$$

where the effective radius of the circular patch is:

$$
a_{\mathrm{eff}}=a\left\{1+\frac{2 h}{\pi a \varepsilon_{r}}\left[\ln \left(\frac{\pi a}{2 h}\right)+1.7726\right]\right\}^{1 / 2} .
$$

For all of the cases examined, the patch radius $a=192.0 \mathrm{~mm}$, and the height of the substrate $h=8.0 \mathrm{~mm}$. As noted, the substrate was assumed to be that of Rogers Duroid 5880, i.e., $\varepsilon_{r}=2.2$. With these parameters, equation (1) gives $f_{\text {res,patch }}=298.9 \mathrm{MHz}$. While it would be uncommon to use a patch antenna at this low of a frequency, it is apparent from equations (1) and (2), that simply by varying its dimensions, one would return to typical patch antenna sizes in the $1-10 \mathrm{GHz}$ range.

We notice immediately that there is no ground plane parameter included in equation (1). In particular, this analytical resonance frequency value does not depend on the ground plane size, but only on the patch size. Thus, the former should have little impact on the impedance matching as well. However, then what effect does the ground plane disk have on the performance of a finite overall-sized patch antenna?

Consider the HFSS model of the coax-fed circular patch shown in Figure 1. The parameters already noted above remained the same. The coax was designed to have a $50 \Omega$ impedance (i.e., the coax had a center conductor whose radius was $1.0 \mathrm{~mm}$, the inner radius of its outer conductor was $2.301 \mathrm{~mm}$, its outer wall was $0.5 \mathrm{~mm}$ thick, and it was 
(a)

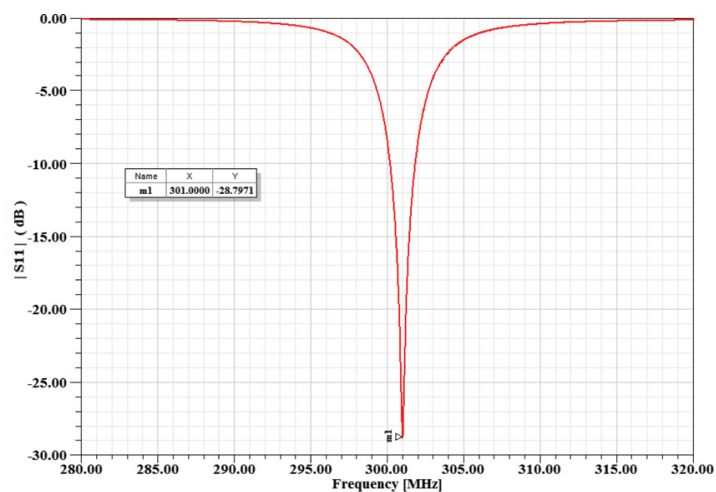

(b)

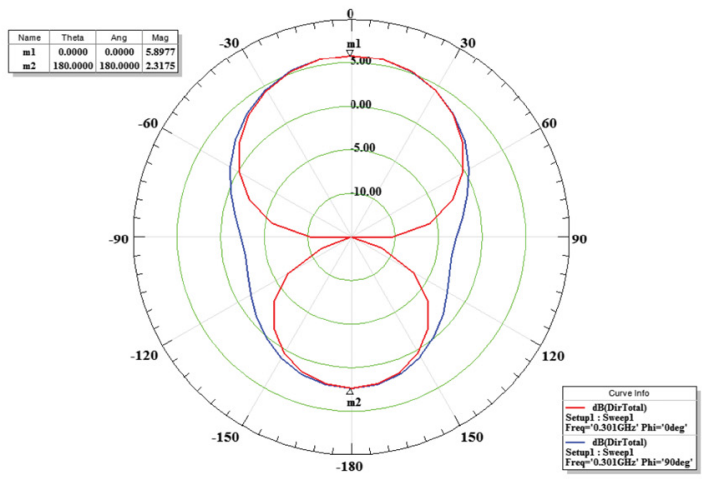

(c)

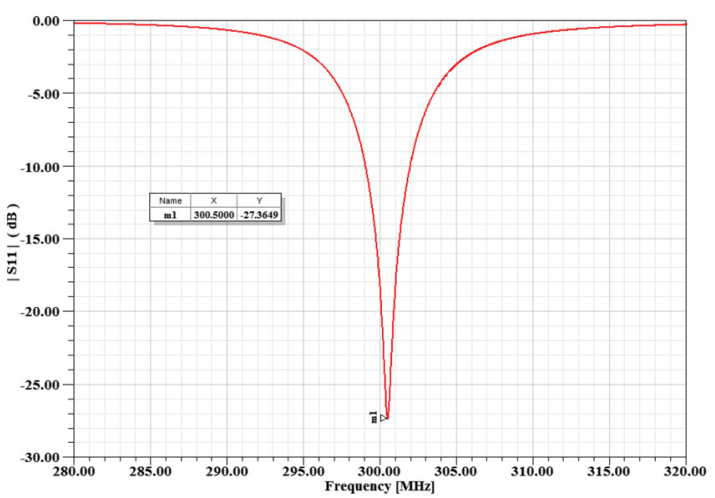

(d)

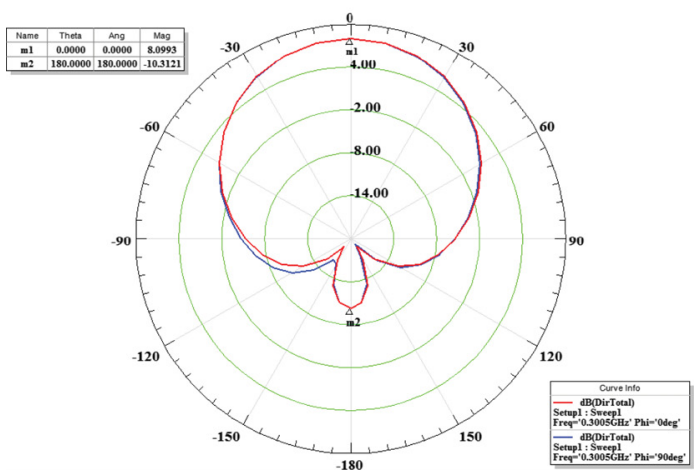

Fig. 2. Patch antenna properties. Ground plane radius $=200$ $\mathrm{mm}$ : (a) $\left|S_{11}\right|$ values versus frequency, (b) directivity pattern at $f_{\text {res }}=301 \mathrm{MHz}$; and ground plane radius $=400 \mathrm{~mm}$ : (c) $\left|S_{11}\right|$ values versus frequency, $(\mathrm{d})$ directivity pattern at $f_{\mathrm{res}}=300.5$ $\mathrm{MHz}$.
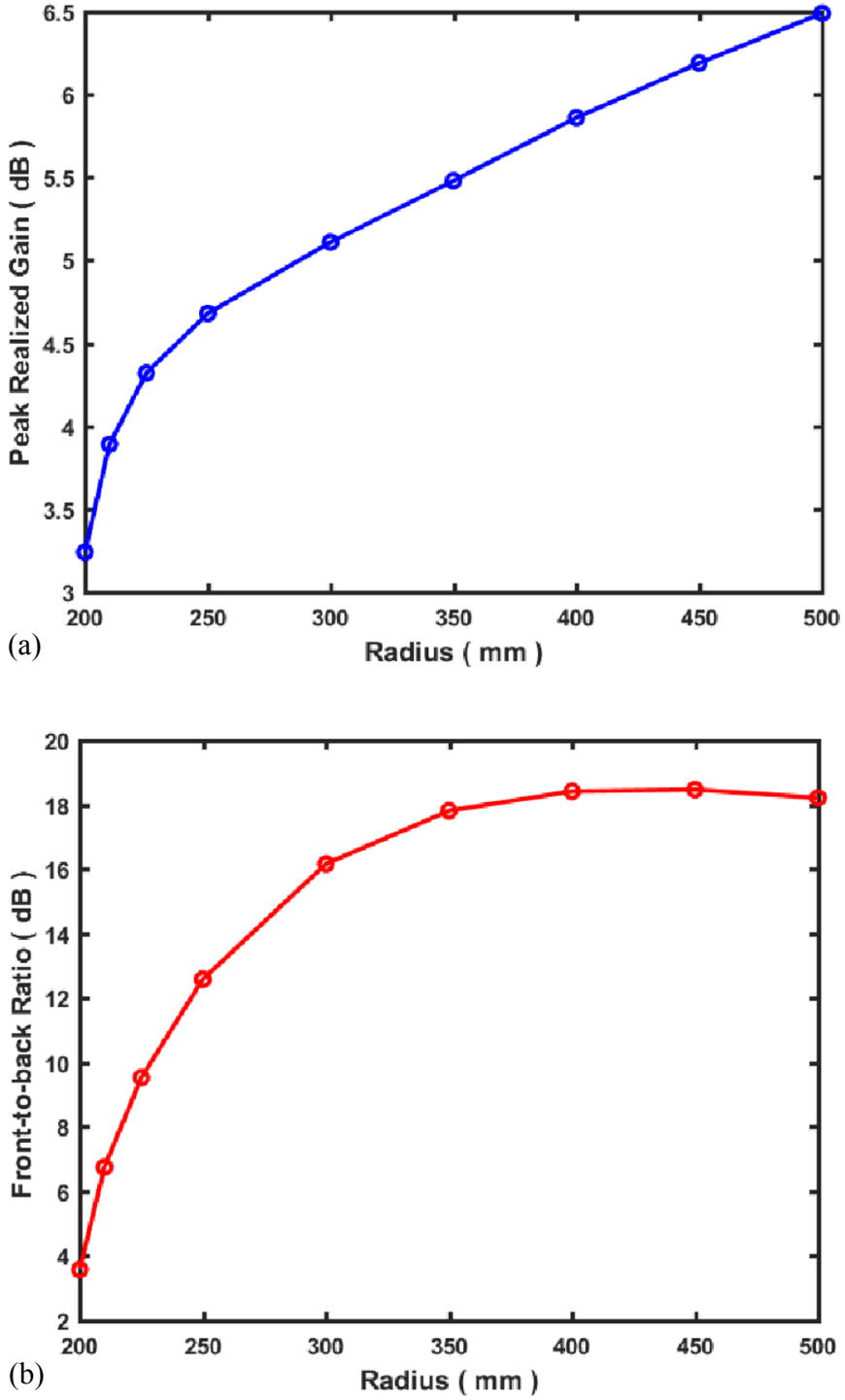

Fig. 3. Patch antenna radiation properties as functions of the ground plane radius. (a) Realized gain and (b) FTBR.

filled with vacuum). The length of the coax was $10.0 \mathrm{~mm}$. The coax feed was offset from the origin along the $-\hat{y}$-axis; this offset distance was adjusted to achieve a resonance. The ground plane disk radius, $r_{\mathrm{gp}}$, was taken to be a variable.

The values of $\left|S_{11}\right|$ versus the source frequency and the directivity pattern at the resonance frequency are given in Figure 2 for the patch antennas with $r_{\mathrm{gp}}=200.0 \mathrm{~mm}$ $\left(f_{\text {res }}=301.0 \mathrm{MHz}\right)$ and $r_{\mathrm{gp}}=400.0 \mathrm{~mm}\left(f_{\mathrm{res}}=300.5 \mathrm{MHz}\right)$. The corresponding offset distances of the coax feed were, respectively, -38.0 and $-52.0 \mathrm{~mm}$ along the $y$-axis. While Figures 2a and c indicate nearly complete matching to the source, the ground plane size had no impact on the level of impedance matching once the offset distance was returned. Figures $2 \mathrm{~b}$ and d clearly demonstrate that the ground plane size has a profound impact on the directivity and the FTBR values. These directivity results support the outcomes of earlier explorations of ground plane effects on circular patch antennas [32-34]. 


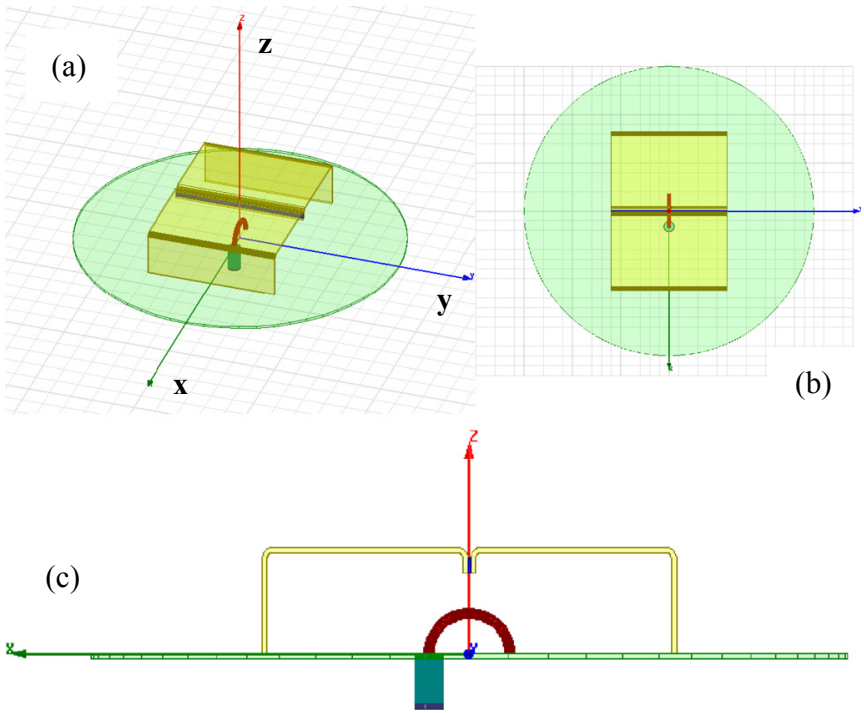

Fig. 4. 3D magnetic EZ antenna. (a) Isometric view, (b) top view, and (c) side view.

One finds, as might be anticipated, that with the ground plane radius nearly the same as the patch radius, the overall structure radiates more like an oversized dipole element. The peak realized gain was $\mathrm{RG}_{\max }=3.24 \mathrm{~dB}$ (radiation efficiency $\mathrm{RE}=84.3 \%$ ) and the $\mathrm{FTBR}=3.58$ $\mathrm{dB}$. In contrast, with a ground plane radius double that of the patch, the typical patch emission properties are obtained, i.e., $\mathrm{RG}_{\max }=5.85 \mathrm{~dB} \quad(\mathrm{RE}=90.7 \%)$ and a $\mathrm{FTBR}=18.41 \mathrm{~dB}$.

To investigate these directivity properties further, the ground plane radius was swept from $200 \mathrm{~mm}(8 \mathrm{~mm}$ larger than the patch $)$ to $500 \mathrm{~mm}\left(\sim \lambda_{\text {free space }} / 2\right)$. The directivity and FTBR values that were obtained numerically are shown in Figure 3. While the resonance frequency for each case was less than $1 \%$ away from $300 \mathrm{MHz}$, it is immediately apparent that the ground plane size controls the directivity and FTBR values. Since the radiation efficiency remains above $90 \%$ when $r_{\mathrm{gp}} \geq 250 \mathrm{~mm}$ and nearly complete matching was maintained, the linear-like increase in the realized gain reflects the well-known relation: $G=4 \pi A / \lambda^{2}$, where the area of the antenna is $A \approx \pi r_{\mathrm{gp}}^{2}$. The FTBR begins to roll-off at the largest values of $r_{\mathrm{gp}}$ because the ground plane disk has become on the order of a wavelength in diameter. Its size allows the appearance of higher order modes which interact with the disk's edge, yielding more back radiation.

While it has a significant impact, it must be noted that the ground plane is not the only factor that influences the directivity of a patch antenna. It is well known that surface waves are present. As they are generated and interact with the edges of the patch and the ground plane, they cause scattering into many directions, leading to other variations in the system's directivity. A variety of approaches have been considered recently to mitigate the effects of these surfaces. They include introducing EBG structures, e.g., [35-37]; modified ground planes and metasurfaces, e.g., [38-40]; and even cloaking elements [41].

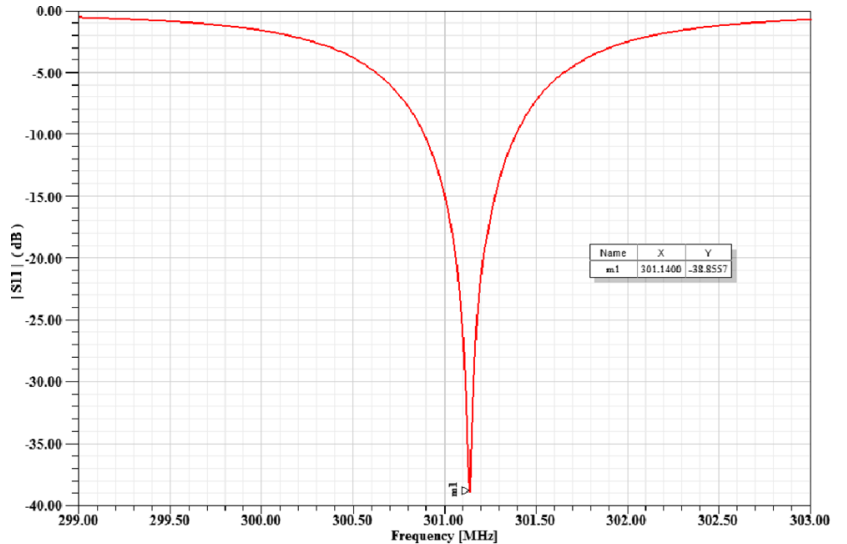

(a)

(b)

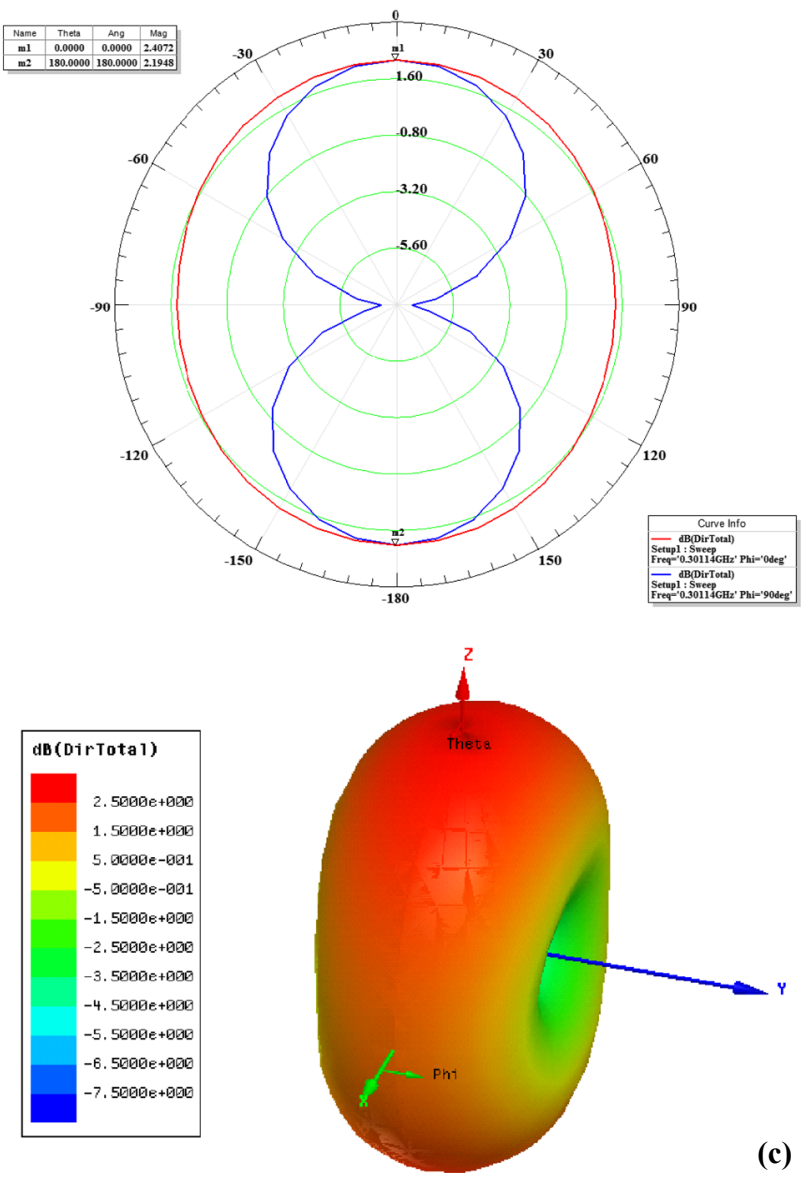

Fig. 5. Electrically small $\left(k a=0.474\right.$ and $\left.r_{\mathrm{gp}}=75.0 \mathrm{~mm}\right) \mathrm{EZ}$ antenna properties. (a) $\left|S_{11}\right|$ values versus frequency, and (b) Eand $\mathrm{H}$-plane directivity patterns and (c) 3D directivity pattern at $f_{\mathrm{res}}=301.14 \mathrm{MHz}$.

\section{3D magnetic EZ antenna}

Since the radiation properties of the considered very lowprofile circular patch antennas can be analyzed as arising from a magnetic current loop, the electrically small 3D magnetic EZ antenna [14,42-44], was selected as the 


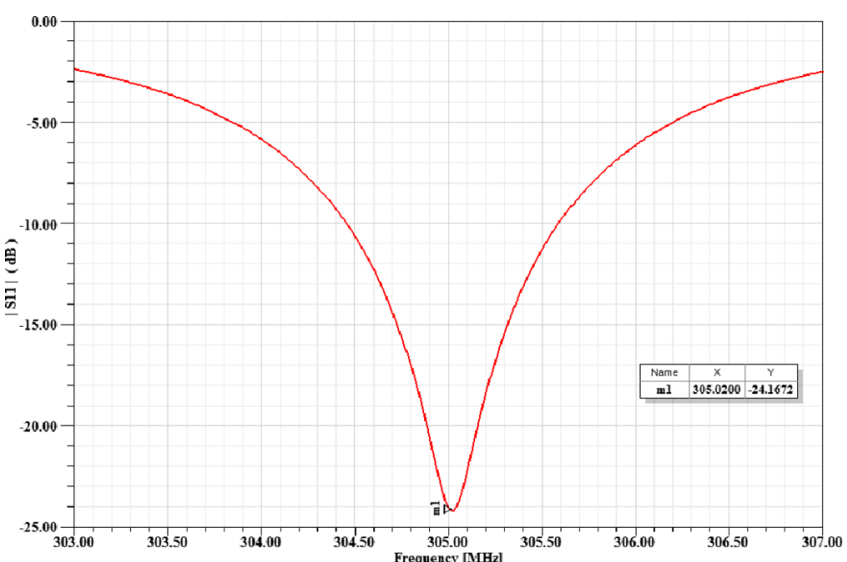

(a)

(b)
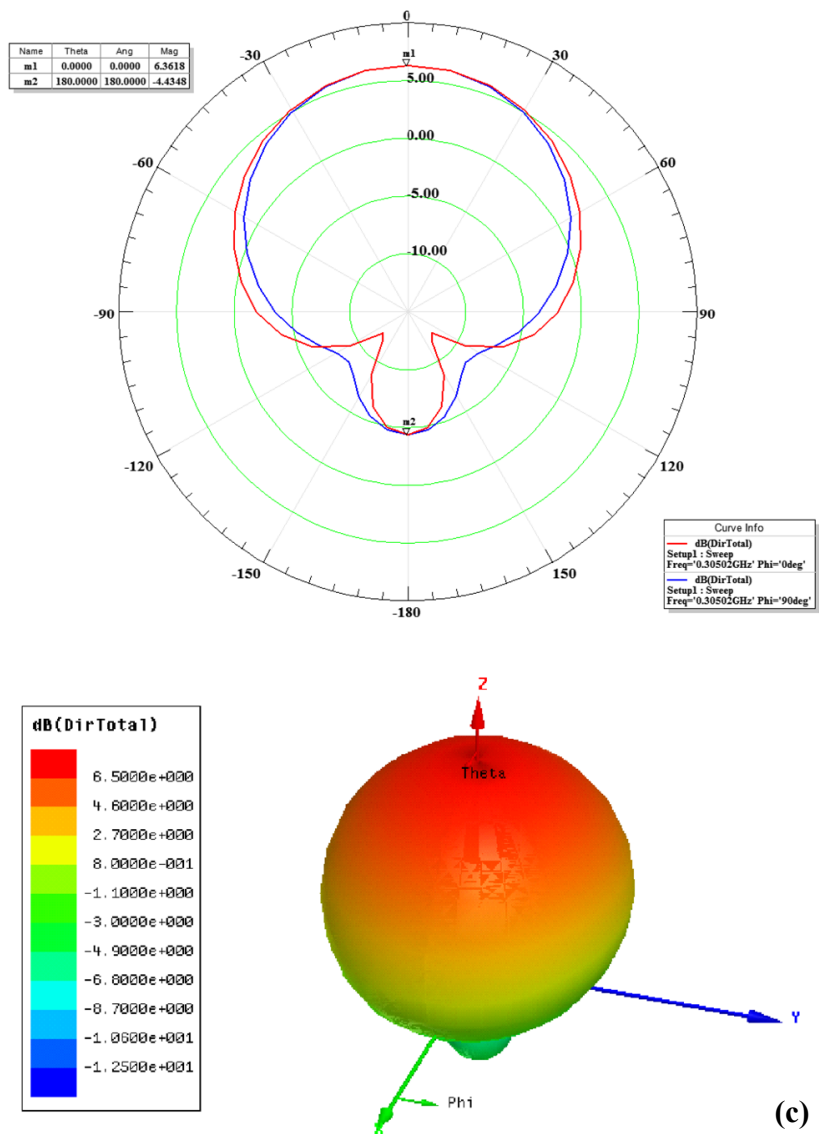

Fig. 6. Electrically large $\left(k a=1.918\right.$ and $\left.r_{\mathrm{gp}}=300.0 \mathrm{~mm}\right) \mathrm{EZ}$ antenna properties. (a) $\left|S_{11}\right|$ values versus frequency, and (b) Eand H-plane directivity patterns and (c) 3D directivity pattern at $f_{\text {res }}=305.02 \mathrm{MHz}$.

NFRP antenna for direct comparisons to the patch antenna directivity results. It radiates as a magnetic dipole over its ground plane.

One of the examined cases is shown in Figure 4. A circular ground plane disk supports a rectangular, extruded capacitively loaded loop (CLL) NFRP element. The coax feed remained the same. The $1.0 \mathrm{~mm}$ center conductor was extruded to form a semi-loop antenna. This driven semi-loop antenna has an $8.0 \mathrm{~mm}$ center radius. The ground plane and the NFRP element were both $1.0 \mathrm{~mm}$ thick copper. The NFRP element is centered with respect to the semi-loop. It has an outside $x$-length of $82 \mathrm{~mm}$ ( $80 \mathrm{~mm}$ length from inside edge to inside edge), an outside $y$-length of $60 \mathrm{~mm}$, and a $21 \mathrm{~mm}$ total height ( $20 \mathrm{~mm}$ from ground plane to bottom face). The rolled edges of the NFRP element all had $1.0 \mathrm{~mm}$ radii. The dielectric in the gap was quartz $\left(\varepsilon_{r}=3.78, \mu_{r}=1.0\right.$ and loss tangent $=0.0)$. It is $0.625 \mathrm{~mm} \times 60 \mathrm{~mm} \times 3.05 \mathrm{~mm}$ in size and rests flush with the copper strips forming the capacitor gap. The ground plane disk has a $75.0 \mathrm{~mm}$ radius.

The $\left|S_{11}\right|$ values as a function of the source frequency, and the $2 \mathrm{D}$ and $3 \mathrm{D}$ directivity patterns are shown in Figure 5. The resonance frequency is $301.14 \mathrm{MHz}$, giving $k a=0.474$, where here " $a=75 \mathrm{~mm}$ ", the ground plane radius, is also the radius of the smallest sphere enclosing the entire antenna. This electrically small antenna has $\mathrm{RE}=$ $88.3 \%, \quad \mathrm{RG}_{\max }=1.54 \quad(1.875 \mathrm{~dB}), \quad$ and $\quad \mathrm{FTBR}=1.05$ $(\sim 0 \mathrm{~dB})$. It clearly radiates as a magnetic dipole oriented along the $y$-axis.

As with the patch antenna, increasing the ground plane disk radius to four times the size: $r_{\mathrm{gp}}=300 \mathrm{~mm}$, one observes that its directive properties are impacted significantly. As shown in Figure 6, the EZ antenna is resonant at $305.02 \mathrm{MHz}$ (a slight $1.3 \%$ blue-shift) and, of course, is now electrically large with $k a=1.918$ (and hence, obviously, has more bandwidth). As shown in Figures $6 \mathrm{~b}$ and c, the larger ground plane has significantly broadened its directivity pattern and dramatically reduced the size of its back-lobe. It performance characteristics include $\mathrm{RE}=94.7 \%, \quad \mathrm{RG}_{\max }=4.08$ $(6.11 \mathrm{~dB})$, and FTBR $=12.01(10.80 \mathrm{~dB})$. As observed in Figure $6 c$, its directivity pattern is now very similar to the patch antenna case whose ground plane is also several times larger than its patch radiator.

To make further comparisons with the patch antenna results, the ground plane size was swept through a variety of values. The FTBR and RG values as functions of $r_{\mathrm{gp}}$ are shown in Figure 7. These values have begun to taper off sooner than those of the patch antenna. As reported previously [42], the currents associated with the EZ antenna are quite confined to the region in the vicinity of the NFRP element. Consequently, the much larger ground plane disk is not being excited efficiently by those currents and, hence, the enhancement of the directivity performance does not continue. On the other hand, the patch antenna never has a chance to be electrically small. Its physics requires a half-wavelength or more in size to achieve a resonance. While more advanced techniques such as the introduction of zero-index [45], epsilon-nearzero [46], or transformation optics based [47] metamaterials may help maximize the overall directivity associated with given effective area, they will not remove the intrinsic ground plane and half-wavelength requirements of the patch antenna. Moreover, their inclusion into the overall structure would most likely increase its physical size to accommodate enough unit cells to achieve the desired material properties in any practical realization. Thus, what can one do to increase the directivity and still be electrically small? 

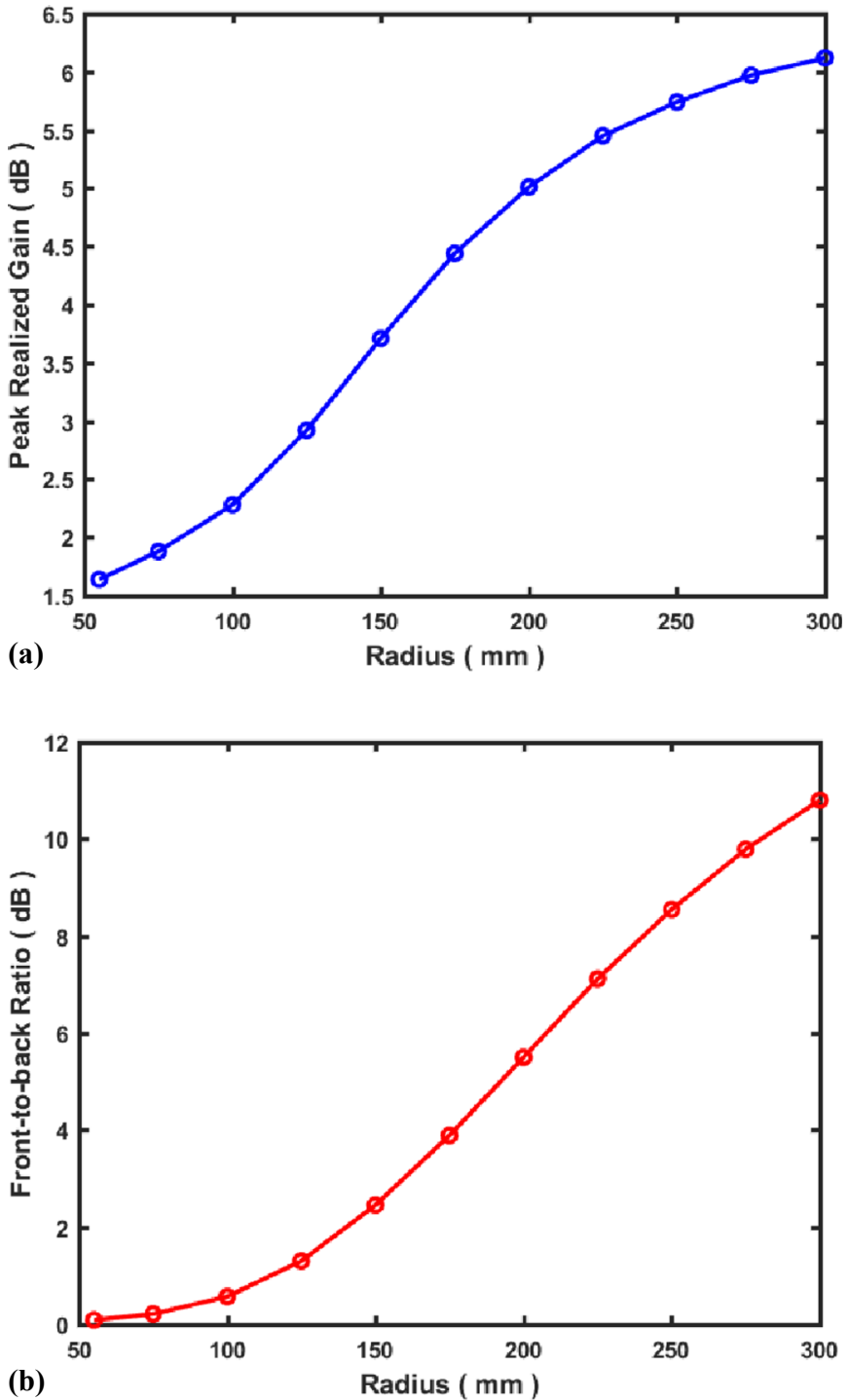

Fig. 7. 3D magnetic EZ antenna radiation properties as functions of the ground plane radius. (a) Realized gain and (b) FTBR values.

\section{Compact array with two NFRP antennas}

While EBG structures (structured ground planes) have been used for back-lobe reduction [9], they too are not electrically small, again by the physical processes used to achieve their properties. On the other hand, recent designs have suggested that Huygens sources may be advantageous, particularly in regards to their FTBR values [20-25]. Consequently, it was explored whether or not the magnetic-based EZ antenna could be augmented with an electric-based NFRP antenna to achieve an electrically small Huygens source. This two-element NFRP antenna array is depicted in Figure 8. The array consists of a rectangular ground plane version of the EZ antenna and a rectangular version of the Egyptian axe dipole (EAD) antenna [14]. The rectangular nature of both antennas and their orthogonal linear polarization behaviors allow them to be densely packed. Each has its own coax feed.

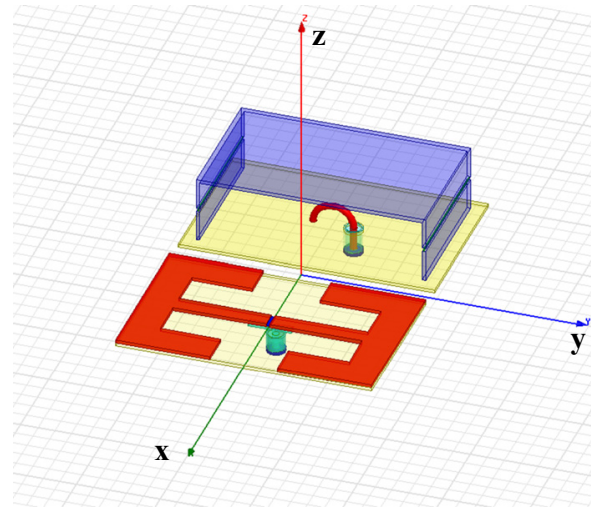

Fig. 8. Compact array consisting of two NFRP antennas, the rectangular EZ antenna and the rectangular EAD antenna.

Consider first the magnetic EZ antenna. As shown in Figure 9, it has no rounded edges; they are simply right angled here. This simplified the simulations. The rounded edges were originally introduced in $[42,43]$ because of the fabrication methods used to construct it. The outside lengths in $x, y$ and $z$ of the CLL NFRP element are (in $\mathrm{mm}$ ) 40.0,68.0, and 20.0, respectively. This means the minimum enclosing sphere radius is $a=44.23 \mathrm{~mm}$. The ground plane was $48 \mathrm{~mm} \times 76 \mathrm{~mm}$, i.e., only $4.0 \mathrm{~mm}$ beyond each side of the CLL. The physical capacitor was replaced with two lumped element capacitors, each with capacitance $C_{\mathrm{CLL}}=20.5 \mathrm{pF}$ and each extending completely across the gaps $(1.0 \mathrm{~cm}$ along the $z$ direction) centered on both vertical segments (sides normal to the $y$-axis). While it again simplified the simulations, this choice allows for frequency agile versions, as demonstrated with the box cage loop antenna version [44].

The ground plane and CLL metal thicknesses remained at $1.0 \mathrm{~mm}$. The coax feed was the same except that its length was now $7.0 \mathrm{~mm}$. The center radius of the wire semiloop was $6.0 \mathrm{~mm}$. The semi-loop and the CLL element were again centered with respect to the coordinate origin. Figure 10 shows several of the simulated performance characteristics. It was resonant at $f_{\text {res }}=299.798 \mathrm{MHz}$, yielding $k a=0.28$. It had $\mathrm{RE}=73.0 \%, \mathrm{RG}_{\max }=1.23$, and $\mathrm{FTBR}=1.0$ at $f_{\text {res }}$. The $3 \mathrm{D}$ directivity pattern clearly indicates that it acts a magnetic dipole oriented along the $x$ axis. The lower $\mathrm{RE}$ value is a consequence of its smaller electrical size.

Next, consider the rectangular EAD antenna. It is shown in Figure 11 along with its dimensions. The dielectric thickness was $0.7874 \mathrm{~mm}$ (31 mils). The thickness of all of the copper strips was $1.0 \mathrm{~mm}$. The center gap in the NFRP element was $1.016 \mathrm{~mm}$ (40 mils). An inductor with $L_{\mathrm{EAD}}=161.0 \mathrm{nH}$ was placed across the gap. The same coax was used, but its length in this case was $4.5 \mathrm{~mm}$. The gap between the end of the coax and the connection to the dipole was $1.5 \mathrm{~mm}$. As shown in Figure 11c, the width of the driven dipole is $\mathrm{DW}=2.0 \mathrm{~mm}$, the same as the diameter of the center conductor of the coax. Its total length along the $y$-axis is $\mathrm{DL}=12.301 \mathrm{~mm}$.

The simulated performance characteristics of the EAD antenna are shown in Figure 12. It was resonant at $f_{\text {res }}=300.378 \mathrm{MHz}$, yielding $k a=0.28$. At $f_{\text {res }}, R E=94.0 \%$, $\mathrm{RG}_{\max }=1.56(1.93 \mathrm{~dB})$, and FTBR $=1.0$. The 3D direc- 
(a)
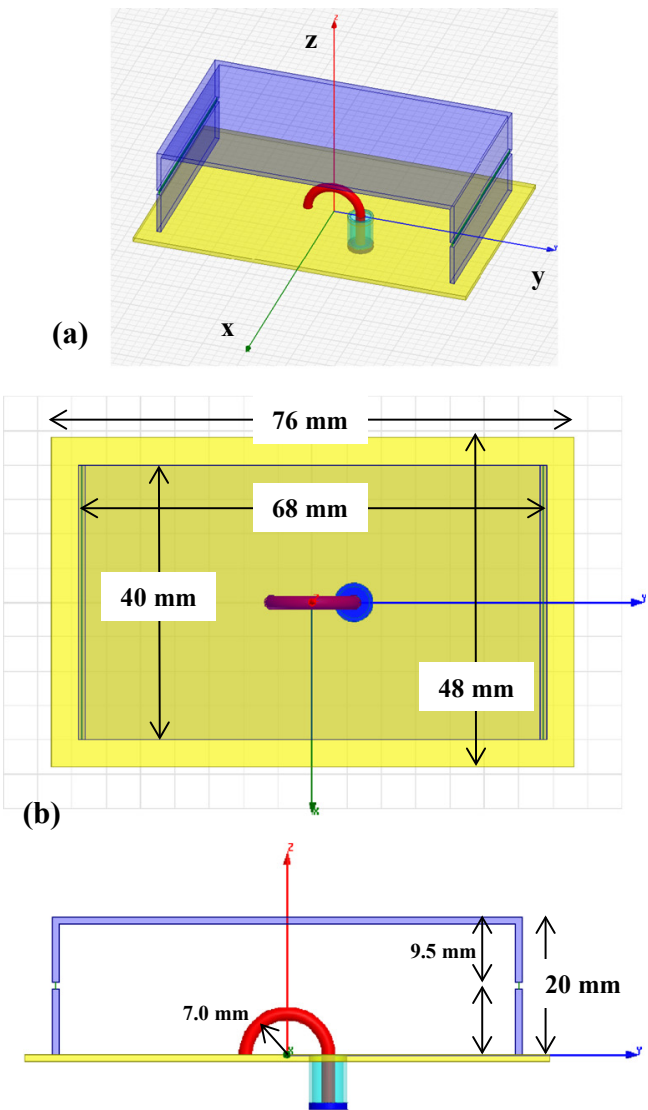

(c)

Fig. 9. Electrically small, rectangular 3D magnetic EZ antenna. (a) Isometric view, (b) top view, and (c) side view.

tivity pattern clearly indicates that the antenna acts as an electric dipole oriented along the $y$-axis. The $\mathrm{RE}$ value is larger than the EZ value; this is a recognized difference between the electric and magnetic antennas when their sizes become very electrically small $[48,49]$.

As shown in Figure 8, these two NFRP antennas were configured into an array. More details of this two-element array are shown in Figure 13. Both the EAD and EZ elements fit within the indicated circle whose radius is $64.5 \mathrm{~mm}$. The initial height of the EAD antenna was set to have the bottom face of its dipole element be at the same height as the bottom face of the ground plane of the EZ antenna. The system is quite low profile given the lowprofile nature of both antennas (note that the EZ antenna is $\sim \lambda / 50$ in height). The antenna pair is also very compact; the centers of the NFRP elements are separated by only $56 \mathrm{~mm}(\sim \lambda / 17.86)$.

Because the main current flow on both NFRP elements is along the $y$-direction, it was expected that there would be strong coupling between the two radiating elements. The initial simulations of this array confirmed this behavior. It was determined that the resonance of the EAD element was more strongly impacted. It was retuned to achieve a good overlap of the resonances of both radiating elements by simply changing the inductor value to $L_{\mathrm{EAD}}=153.95 \mathrm{nH}$ and increasing the total length of the driven dipole to $12.801 \mathrm{~mm}$ (individual pieces increased by $0.25 \mathrm{~mm}$ ). The

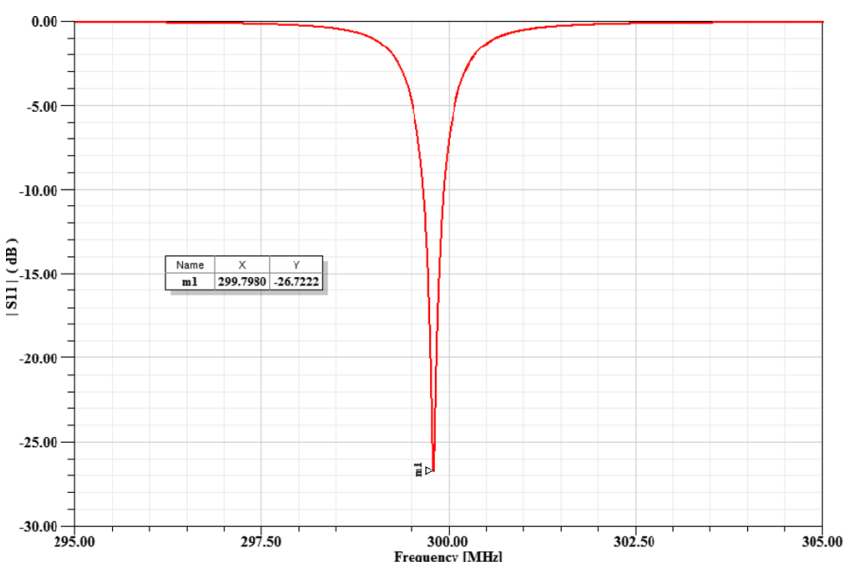

(a)
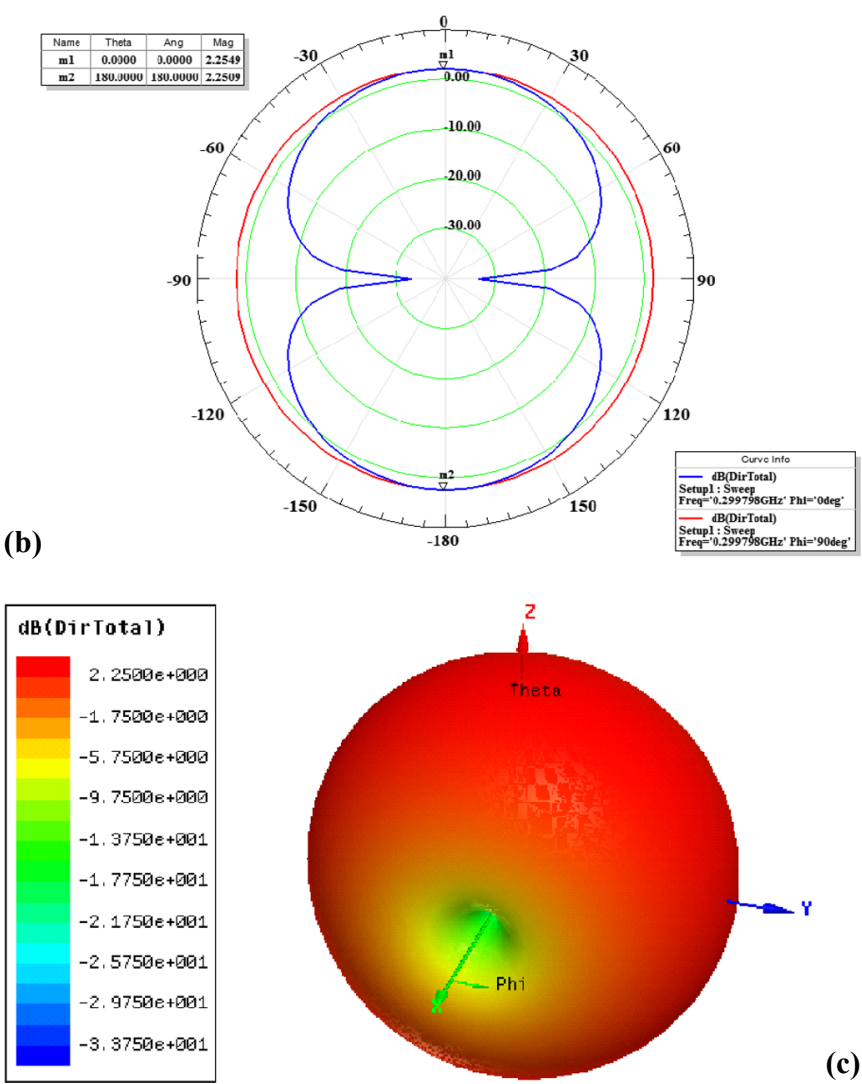

(c)

Fig. 10. Electrically small $(k a=0.28)$ EZ antenna properties. (a) $\left|S_{11}\right|$ values versus frequency, and (b) E- and H-plane directivity patterns and (c) $3 \mathrm{D}$ directivity pattern at $f_{\mathrm{res}}=299.798 \mathrm{MHz}$.

decrease in the lumped element inductor value upshifts the EAD resonance frequency. The increase in the length of the driven dipole provides a small increase in inductance to retune the input impedance matching. By itself, this version of the EAD has $f_{\text {res }}=306.83 \mathrm{MHz}$. This behavior makes sense physically because the EZ NFRP element presents a large inductance to the EAD system. Furthermore, because it is primarily a magnetic field driven and because of its orthogonal orientation to the NFRP element, the EZ system is affected little by the presence of the EAD elements. 


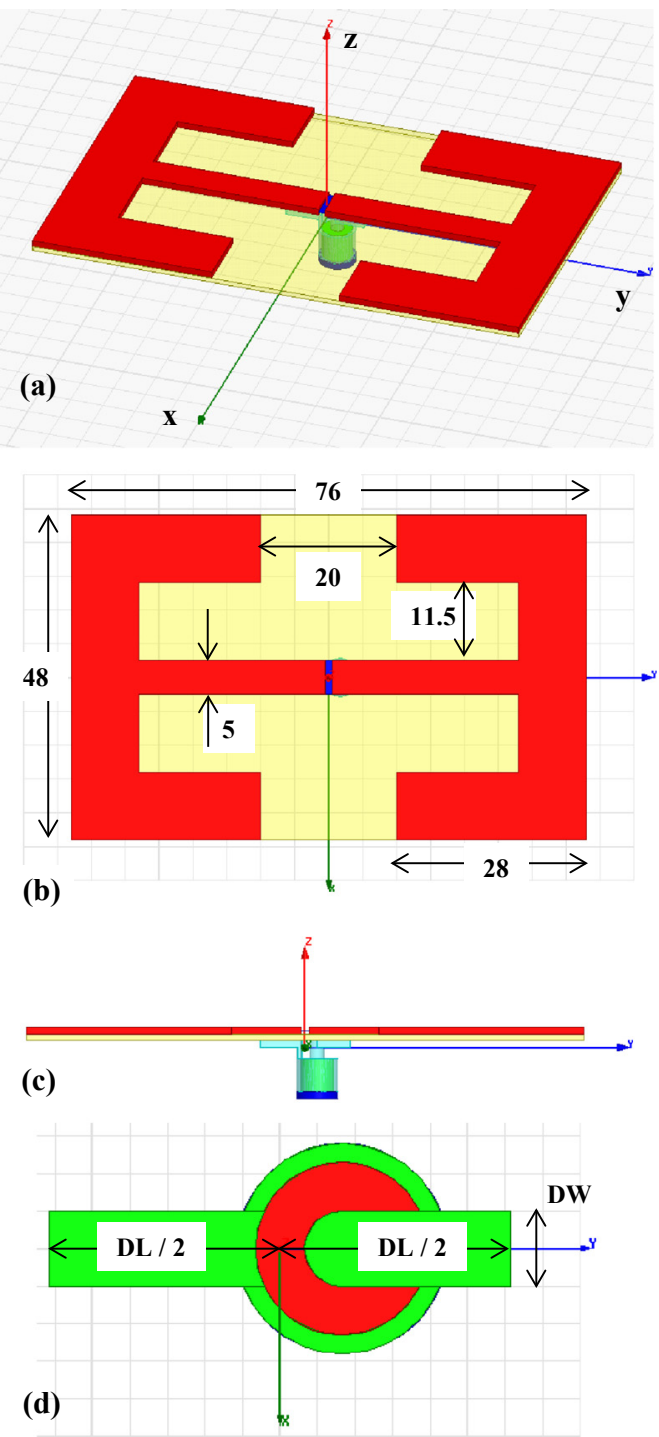

Fig. 11. Electrically small rectangular EAD antenna. (a) Isometric view, (b) top view of NFRP, (c) side view, and (d) top view of coax-fed dipole. All indicated distances are in millimeters $(\mathrm{mm})$.

The simulated performance characteristics of this array are shown in Figures 14 and 15. In Figure 14a, it is observed that the resonances of the two NFRP antennas overlap nicely. The EZ antenna is resonant at $f_{\text {res }}=$ $299.79 \mathrm{MHz}$; the EAD antenna at $f_{\text {res }}=300.09 \mathrm{MHz}$. In Figure 14b, one finds that the radiation efficiency varies slightly with frequency and has its peak at $299.75 \mathrm{MHz}$, slightly below the EZ resonance frequency. Figures 14c and $\mathrm{d}$ give, respectively, the FTBR values and the accepted and total radiated powers. The maximum FTBR ratio, $2505(34.0 \mathrm{~dB})$, occurs at $299.07 \mathrm{MHz}$. Unfortunate-

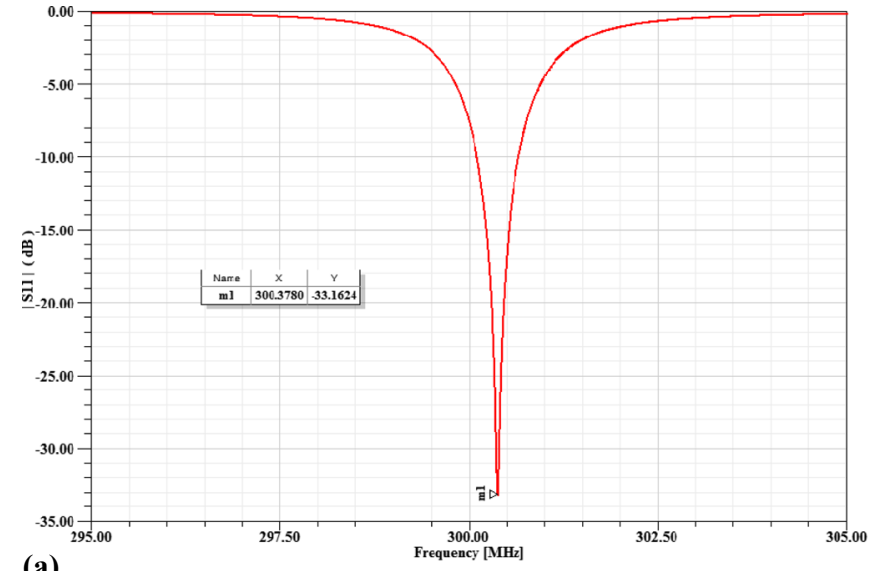

(a)
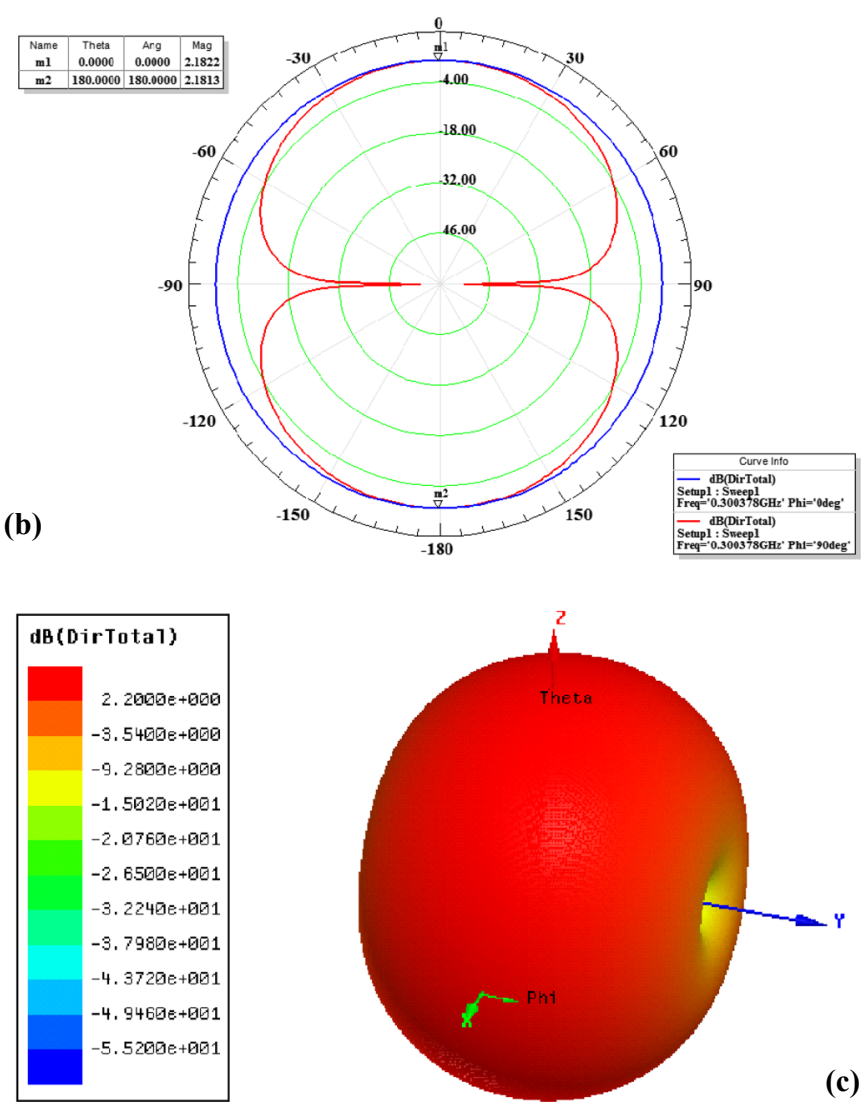

Fig. 12. Electrically small $(k a=0.28)$ EAD antenna properties. (a) $\left|S_{11}\right|$ values versus frequency, and (b) E- and H-plane directivity patterns and (c) $3 \mathrm{D}$ directivity pattern at $f_{\text {res }}=$ 300.378 MHz.

ly, at that frequency the accepted and total radiated powers for a $1.0 \mathrm{~W}$ input power are, respectively, only 0.23 and $0.22 \mathrm{~W}$. On the other hand, the FTBR is only 2.62 $(4.18 \mathrm{~dB})$ at $299.75 \mathrm{MHz}$. Moreover, the maximum radiated power, $0.78 \mathrm{~W}$, occurs next to the $\mathrm{EAD}$ resonance frequency at $300.1 \mathrm{MHz}$, where $\mathrm{RE}=96.3 \%$ and $\mathrm{FTBR}=$ $1.43(1.55 \mathrm{~dB})$.

Note that while the frequency at which the radiation efficiency is maximum was found near the EZ resonance frequency, the frequency at which the total radiated power is maximum was found near the EAD resonance frequency. 


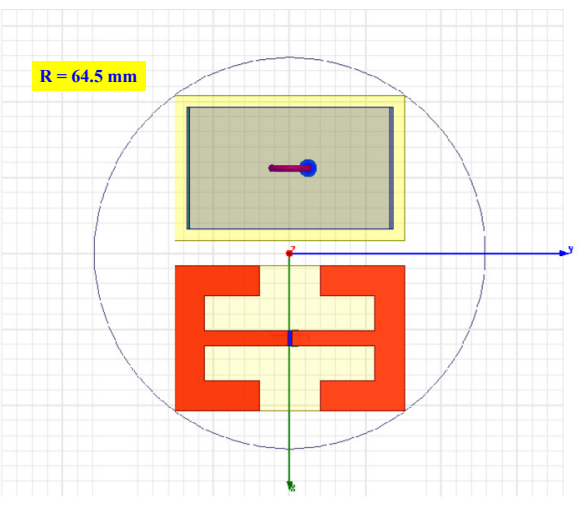

(a)

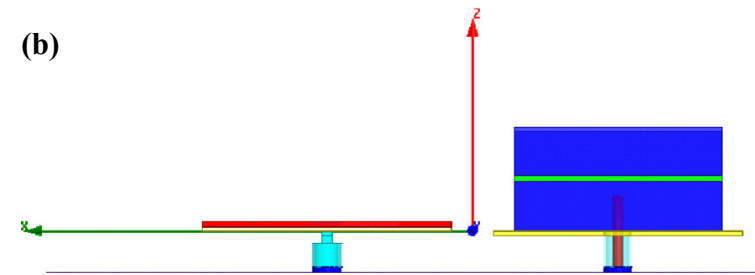

Fig. 13. Electrically small array with two NFRP antennas. (a) Top view and (b) side view.

This behavior arises because when they are driven separately, the EAD element is a more efficient radiator in the array than the EZ element is.

The directivity patterns at $299.07 \mathrm{MHz}$ where the maximum FTBR value occurs are shown in Figure 15. Figure 15a clearly shows the system generates a cardioid pattern. As desired, the electric and magnetic dipoles combine to yield a Huygens source behavior. Unfortunately, this Huygens source behavior does not occur at the peak of the total radiated power. Nonetheless, the $22 \%$ output power with FTBR $=34 \mathrm{~dB}$ may be acceptable for some applications.

A large number of attempts were made to cause the maximum FTBR and total radiated power frequencies to overlap significantly when both elements were driven. This notably included shifting the phase between the two elements through their sources. While the latter did provide a means to shift the max FTBR frequency as desired, the actual total power radiated was reduced even further when it overlapped the maximum total radiated power frequency. Detailing the current behaviors, it was found that as the frequencies were made to overlap, the currents became more out of phase to achieve the high FTBR. Consequently, it was decided to try shorting out one element, as one would for achieving high directivity with an end-fire array [4-8].

Again, metamaterials and other types of elements could have been introduced in between the EAD and EZ radiating elements to reduce any mutual coupling effects. This approach to decoupling between radiating elements has been studied in many configurations, e.g., [50-66]. However, because any of these implementations would require additional area/volume, they would substantially increase the overall size of the system and, hence, would nullify the intended electrically small result. The miniaturization advantages of the metamaterial-inspired NFRP elements is thus further demonstrated.

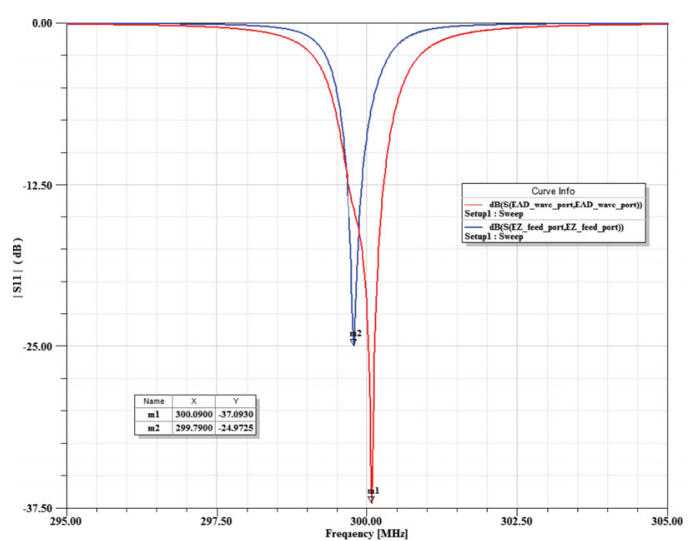

(b)

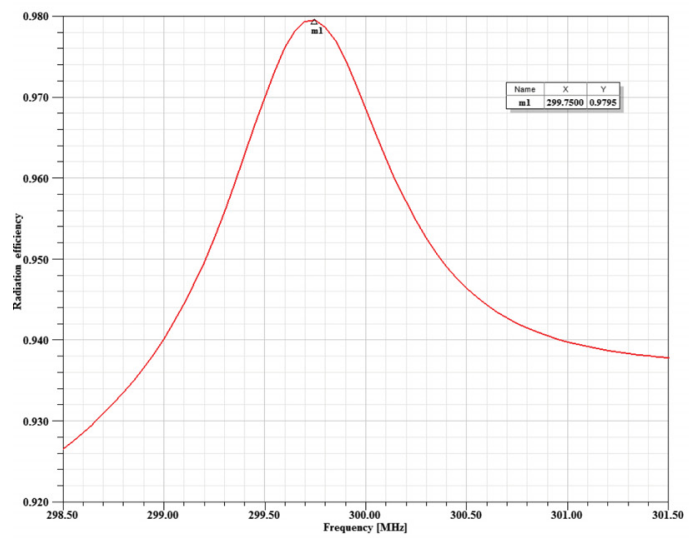

(c)

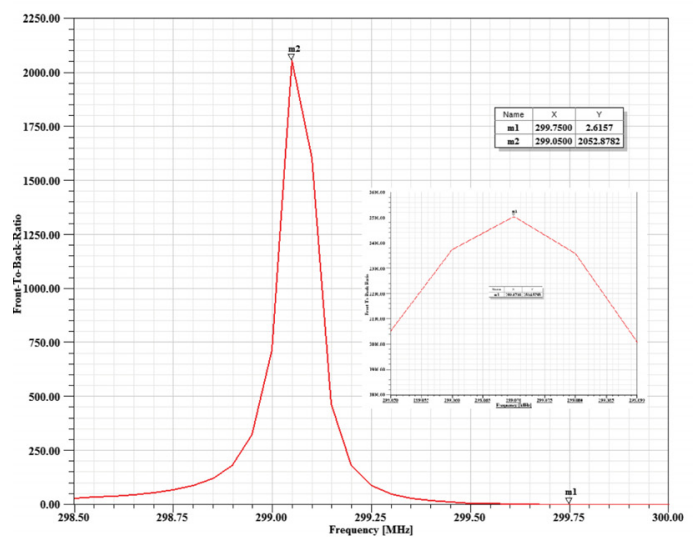

(d)

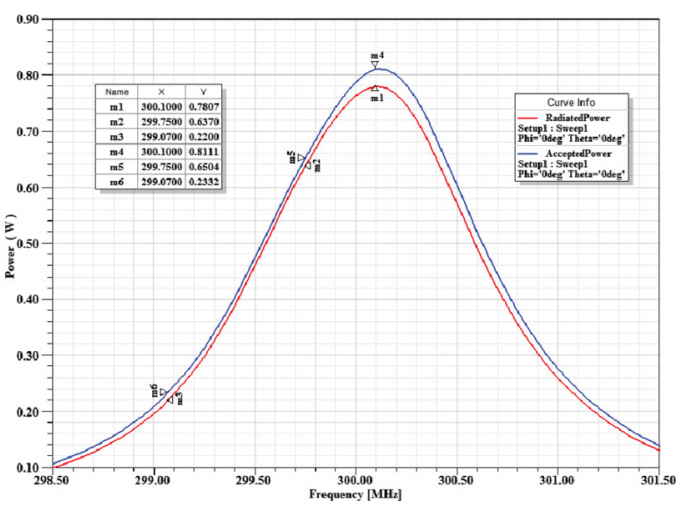

Fig. 14. Array with two NFRP antennas with both elements driven. As functions of the source frequency, the values of (a) | $S_{11} \mid$, (b) radiation efficiency, (c) FTBR, and (d) accepted and total radiated powers. 

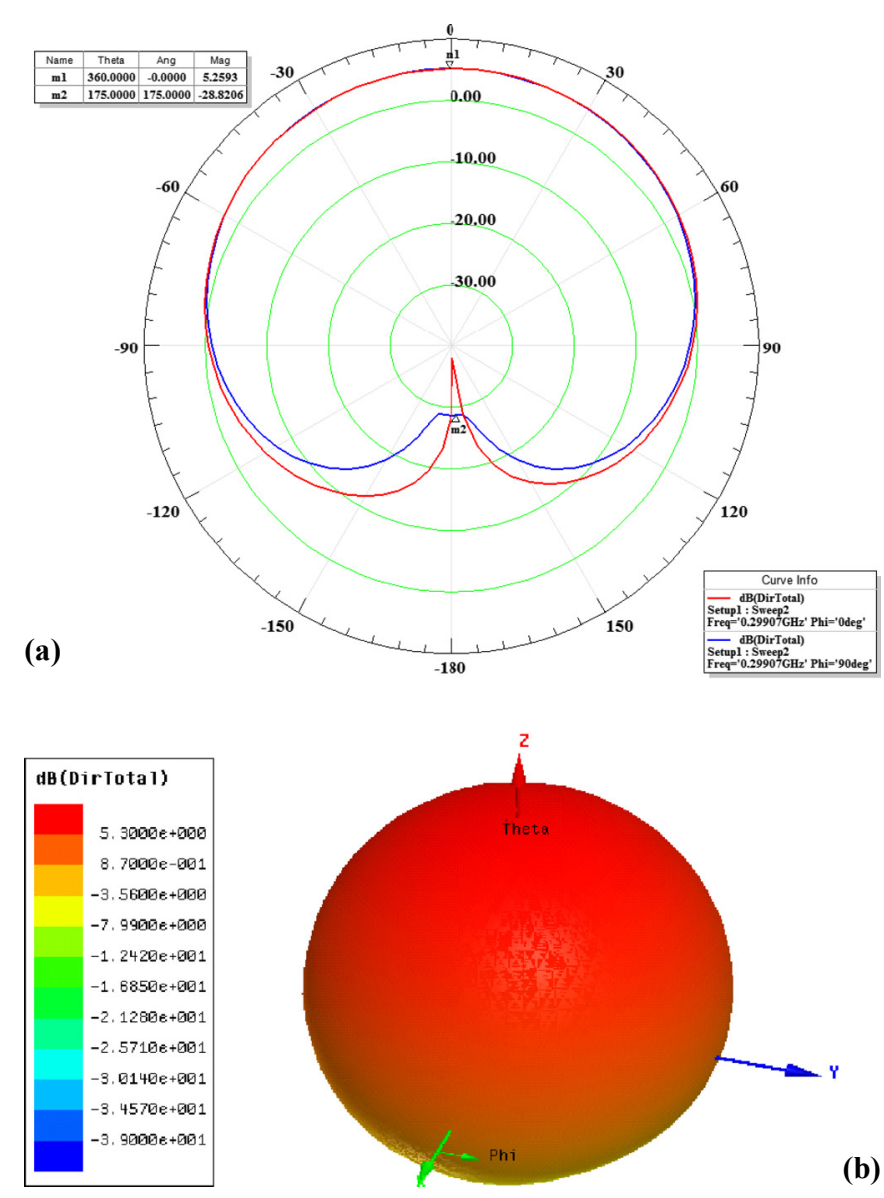

(b)

Fig. 15. The (a) E- and H-plane, and (b) 3D directivity patterns for the array with two NFRP antennas with both elements driven at the frequency $299.07 \mathrm{MHz}$ at which the peak FTBR value occurs.

\section{Compact array of two NFRP antennas, only EAD driven}

Because of the strength of the EAD antenna's emissions in comparison with those of the EZ antenna, it was decided to simply short out the source element of the EZ antenna in the HFSS model. This configuration is shown in Figure 16. Note that the top view is identical to the one shown in Figure 14a. The elements were retuned slightly to achieve good impedance matching. In particular, in comparison with the original two-element array values, setting $C_{\mathrm{CLL}}=20.4 \mathrm{pF}$ (slight increase in EZ resonance frequency), semi-loop radius to be $5.72 \mathrm{~mm}$ (slight decrease in inductive reactance), the gap in the arms of the EAD being increased from 20 to $22 \mathrm{~mm}$ (decrease in capacitive reactance), $L_{\mathrm{EAD}}=155.6 \mathrm{nH}$ (decrease in EAD resonance frequency, and a $11.801 \mathrm{~mm}$ dipole total length (slight increase in capacitive reactance) produced the desired design. Additionally, the coax length of the EAD antenna was raised $4.4994 \mathrm{~mm}(8.9994 \mathrm{~mm}$ total length) to align the phase centers of both of the radiating NFRP elements.

The simulated performance characteristics of this array of two NFRP antennas with only the EAD element driven are shown in Figures 17 and 18. Figure 17a indicates that a

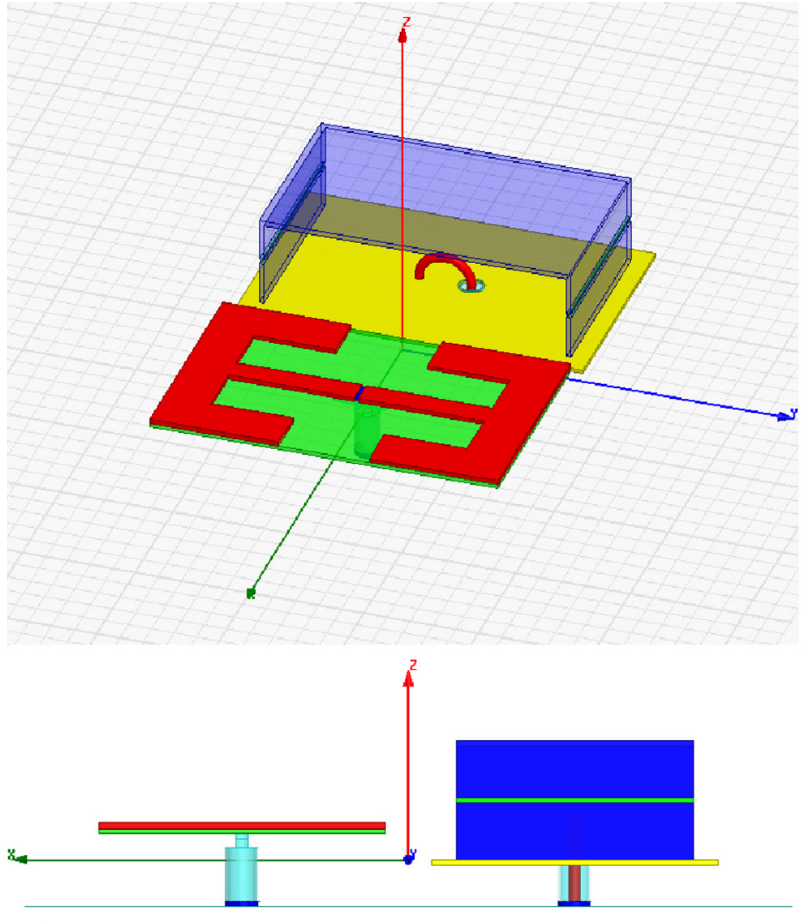

(a)

(c)

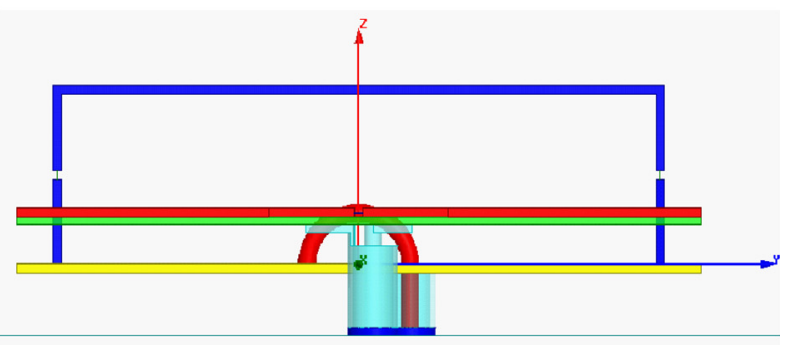

(b)

Fig. 16. Array of two NFRP antennas with only the EAD element driven. (a) Isometric view, (b) $y$-axis side view, and (c) $x$ axis side view.

good overlap of the resonances of both elements was achieved. From Figure $17 \mathrm{~b}$ and $\mathrm{c}$ one finds that the frequencies at which the peak values of the radiation efficiency and the FTBR occur now coincide at $301.154 \mathrm{MHz}$. The inset sub-plot in Figure 17c is a finer resolution of the FTBR values near its peak. The peak FTBR value is $510.54(27.0 \mathrm{~dB})$ where $\mathrm{RE}=95.31 \%$. At that frequency, the accepted and total radiated powers are, respectively, 0.949 and $0.904 \mathrm{~W}$. The $2 \mathrm{D}$ and $3 \mathrm{D}$ directivity patterns at $301.154 \mathrm{MHz}$ are shown in Figures 18a and b, respectively. They clearly demonstrate that the desired cardioid pattern has been generated by this array.

Using the HFSS model to determine the smallest enclosing sphere, it is found that its radius was in fact $a=64.5 \mathrm{~mm}$. This means $k a=0.407$ at the peak FTBR frequency. Consequently, this NFRP antenna array is indeed electrically small.

While one finds the FTBR peak occurs where there is a slight dip in the accepted and total radiated powers, the outcomes indicate that the choice to drive one element and short the other has overcome the negative effects of the coupling and out-of-phase currents that occurred with both elements being driven. On the other hand, it has been 
(a)
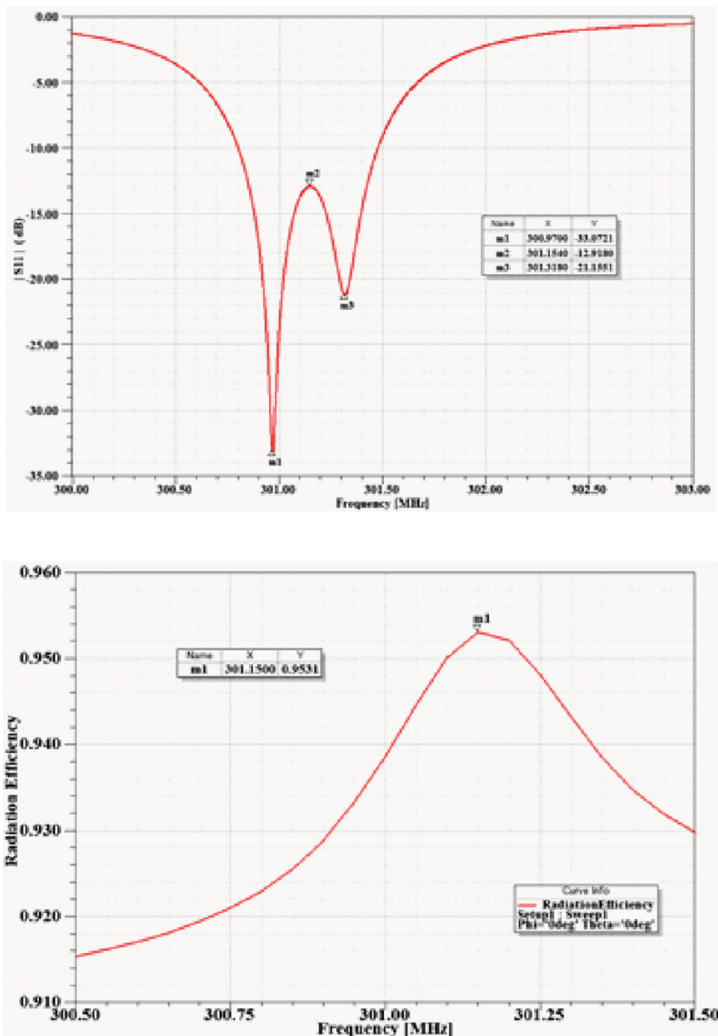

(b)

(c)

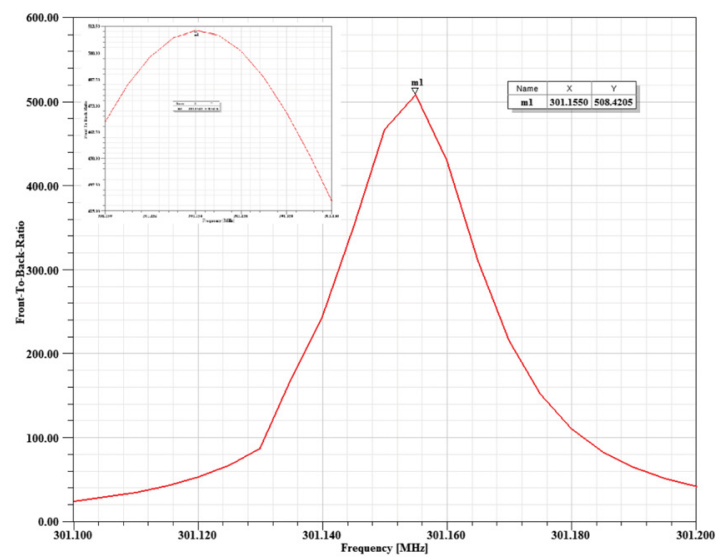

(d)

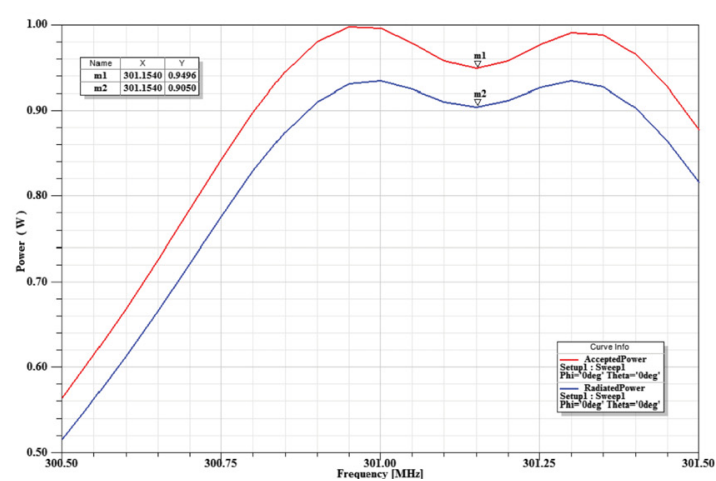

Fig. 17. Array with two NFRP antennas with only the EAD element driven. As functions of the source frequency, the values of (a) $\left|S_{11}\right|$, (b) radiation efficiency, (c) FTBR, and (d) the accepted and total radiated powers.

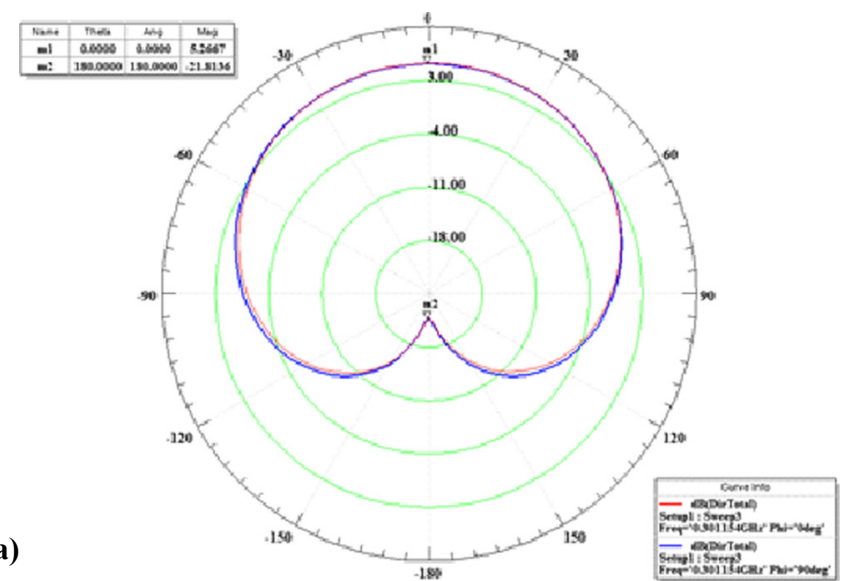

(b)
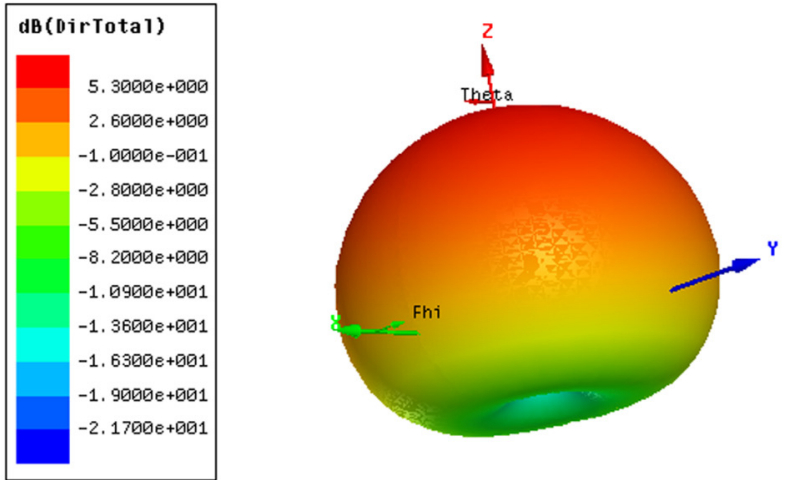

Fig. 18. The (a) E- and H-plane, and (b) 3D directivity patterns for the array with two NFRP antennas with only the EAD element driven at the frequency $301.154 \mathrm{MHz}$ at which the peak FTBR value occurs.

determined that there is a definite tradeoff between the FTBR peak value and the total radiated power. To force a further increase of the former causes the latter to decrease. Again, this behavior is associated with the current flows on these tightly coupled resonators.

\section{Multiple NFRP element Huygens source antenna}

The previously discussed cases have provided further insights into the electrically small, low-profile broadside radiating, Huygens source antennas [22-24]. The configuration of interest is shown in Figure 19. It is a smaller, thinner design than reported in [22] and emphasizes just how electrically small the Huygens antenna can be and still produce the desired cardioid pattern.

The antenna system consists of a driven dipole antenna printed on the bottom face of a circular Rogers 5880 Duroid sheet that is $0.7874 \mathrm{~mm}$ (31 mils) thick and supports layers of $0.017 \mathrm{~mm}$ thick $\left(\frac{1}{2} \mathrm{oz}\right)$ copper. The coax cable has been adjusted slightly to match the design in [22]. The inside surface of the outer wall is now at $2.238 \mathrm{~mm}$ and its length was taken to be $30.0 \mathrm{~mm}$. The gap between the end of the coax and the bottom of the printed dipole antenna was $3.0 \mathrm{~mm}$. The total dipole length was DL $=82.0 \mathrm{~mm}$ and its width remained $2.0 \mathrm{~mm}$. The source side of this printed 
(a)
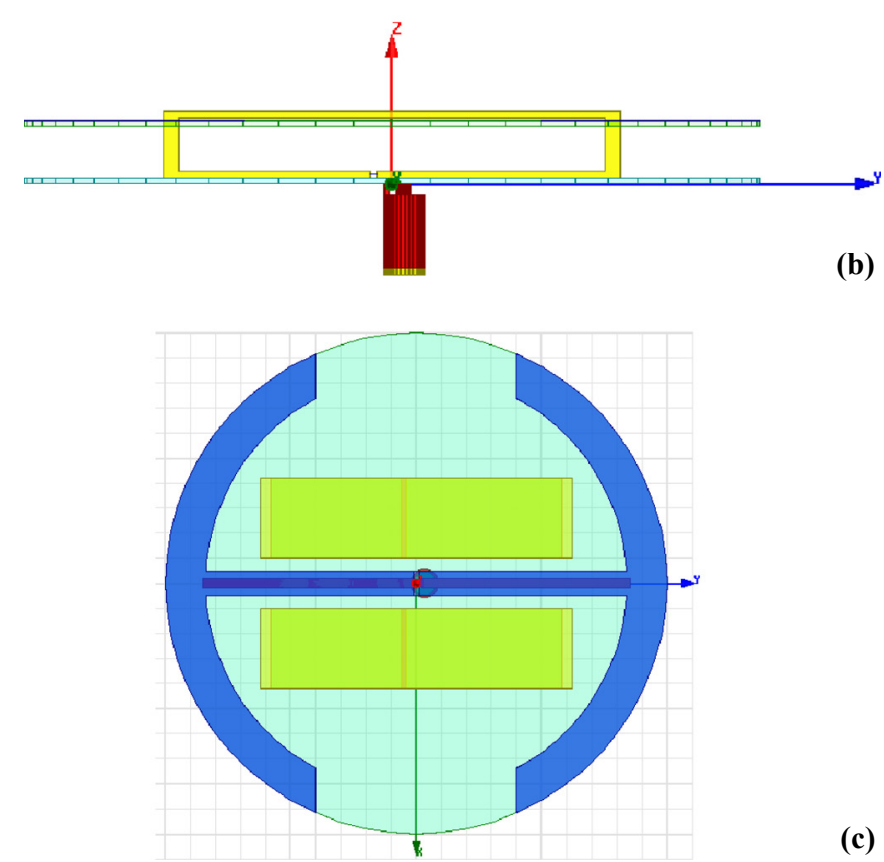

(c)

Fig. 19. Low profile NFRP Huygens source antenna. (a) Isometric view, (b) $x$-axis side view, and (c) $z$-axis top view.

dipole lies on the $x y$-plane. The electric component was an EAD NFRP element; the magnetic NFRP elements were two CLLs.

The EAD NFRP element is now circular in shape. Again, this is a space issue choice. The circular design better fills the enclosing sphere and will generally yield the smallest $k a$ value. It is printed on the top of a second Duroid sheet. The bottom of this second sheet is $7.92 \mathrm{~mm}$ from the top face of the dipole (at $z=7.937 \mathrm{~mm}$ ). Its radius is $50.0 \mathrm{~mm}$, its center strip width is $5.0 \mathrm{~mm}$, its arc width is $5.0 \mathrm{~mm}$, and the gaps in its arc are $20.0 \mathrm{~mm}$. It is oriented with its center strip along the $y$-axis. The inductor is placed across a $1.016 \mathrm{~mm}$ (40 mil) gap centered about $y=0$. The inductor value is $L_{\mathrm{EAD}}=103.5 \mathrm{nH}$.

Each CLL is $16.0 \mathrm{~mm}$ long along the $x$-axis, $62.0 \mathrm{~mm}$ long along the $y$-axis, and $10.0 \mathrm{~mm}$ long along the $z$-axis. The CLLs lie on top of the source substrate. Thus, the entire height of the antenna is $10.8044 \mathrm{~mm}$. Both horizontal strips are $1.0 \mathrm{~mm}$ thick in the $z$-direction; both end, vertical strips are $2.0 \mathrm{~mm}$ thick in the $y$-direction. Both center lines

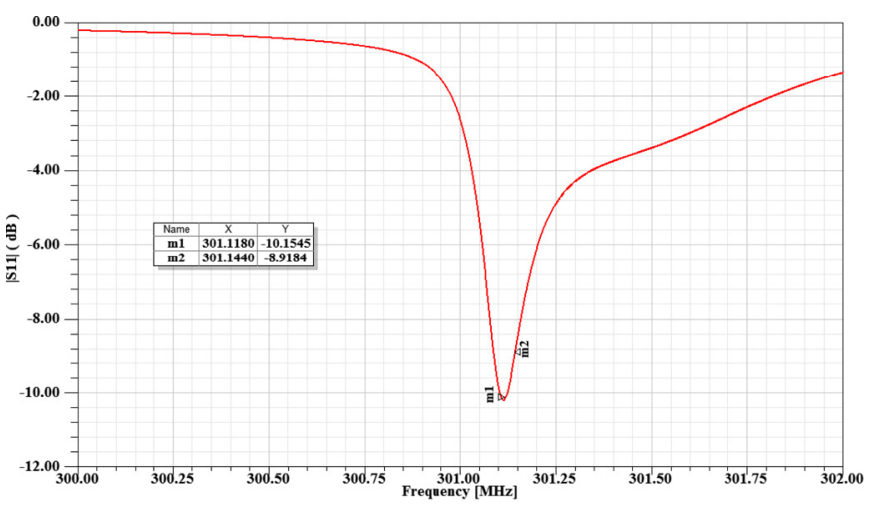

(a)

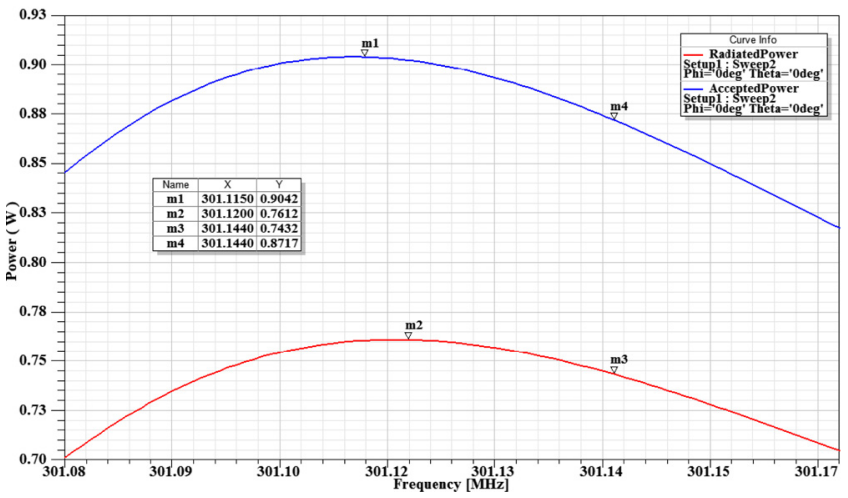

(b)

Fig. 20. Low profile, NFRP Huygens source antenna performance characteristics as functions of the source frequency. The values of (a) $\left|S_{11}\right|$ and (b) the accepted and total radiated powers.

of the CLL elements are offset from the $y$-axis by $13.0 \mathrm{~mm}$. Both capacitor gap centers are offset from the origin along the $y$-axis by $-2.5 \mathrm{~mm}$. The capacitance $C_{\mathrm{CLL}}=10.6 \mathrm{pF}$.

The simulated performance characteristics of this multiple NFPR element antenna are shown in Figures 2022. Figure 20 indicates that a good overlap of the resonances of both NFRP elements was achieved. From Figure 20b one finds that the peak values of the total accepted and radiated power for the $1.0 \mathrm{~W}$ input, are 0.904 and $0.743 \mathrm{~W}$, respectively; and they occur at the frequencies 301.115 and $301.120 \mathrm{MHz}$. Consequently, using the latter frequency, $f_{\mathrm{RP} \text {,peak }}$, the antenna is electrically small with $k a=0.316$ and very low profile (height $=\lambda_{\mathrm{RP} \text {,peak }} /$ 92.15).

The FTBR values as a function of the source frequency are given in Figure 21. The peak FTBR value, 1078.6 $(30.3 \mathrm{~dB})$, occurs at $f_{\text {FTBR peak }}=301.145 \mathrm{MHz}$. The FTBR value at the peak radiated power frequency is 114.6 ( $20.6 \mathrm{~dB})$. The radiation efficiency values at both of these frequencies are $84.34 \%$ and $85.30 \%$, respectively. The slight shift $(0.025 \mathrm{MHz})$ of the FTBR peak frequency from the radiated power peak frequency illustrates how sensitive the phase centering between the electric and magnetic NFRP elements becomes in this strong resonant coupling environment. Nevertheless, very interesting values were obtained for the total radiated power and FTBR at both frequencies. Moreover, it must be noted again that if one 


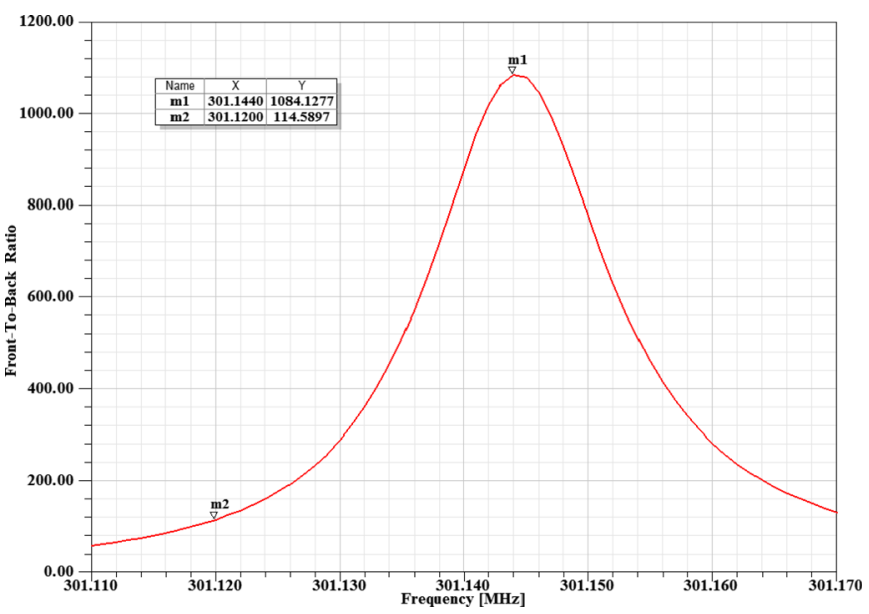

Fig. 21. Low profile, NFRP Huygens source antenna's FTBR values as a function of the source frequency.

retunes the NFRP elements to achieve a higher FTBR, it comes at the cost of less radiated power. Finally, note that despite the presence of a long coax, which would be the case in typical measurement scenarios and could produce a large back-lobe, the electrically small broadside radiating Huygens source effectively emits most of its power into a preferred hemisphere with the desired cardioid pattern.

Even though this quite electrically small, broadside radiating Huygens source system exhibits some sensitivity to its design parameters, slightly larger related designs have been successfully fabricated and measured, confirming the concepts [23]. Moreover, even though a very narrow bandwidth occurs, which arises from the required overlap of two high $Q$ resonators (i.e., the electrically small NFRP elements), it has been demonstrated that by introducing appropriate non-Foster reactances into the NFRP elements, interesting bandwidths can be obtained, while maintaining the desired Huygens source behavior [24]. Almost as low in profile as the patch antenna and lower in profile than the 3D magnetic EZ antenna, these Huygens source designs offer superior directivity performance in a significantly smaller package.

It is interesting to speculate that in the future one might be able to impregnate the essentially dispersionless (at these UHF frequencies), low-loss Teflon substrate in the Duroid sheets with combinations of passive and active core-shell nanoparticles, e.g., [67-72], to create dispersion-tailored macroscopic metamaterial substrates or metasurfaces to enhance the impedance bandwidth of these metamaterialinspired antennas as suggested in [73]. The resulting metastructures could conceivably replace the non-Foster circuits and their complexities to enhance the bandwidth performance of electrically small antennas. They await more advanced nanotechnology fabrication techniques which may become available in the future. On the other hand, one may be able to design and build active lower frequency metastructures with the desired dispersion-tailored characteristics, as well as the antennas themselves, in the near-term using advanced manufacturing methods. This design-torealization approach is currently being investigated through the UK's SYMETA research program led by Prof. J.C. Vardaxoglou at Loughborough University.

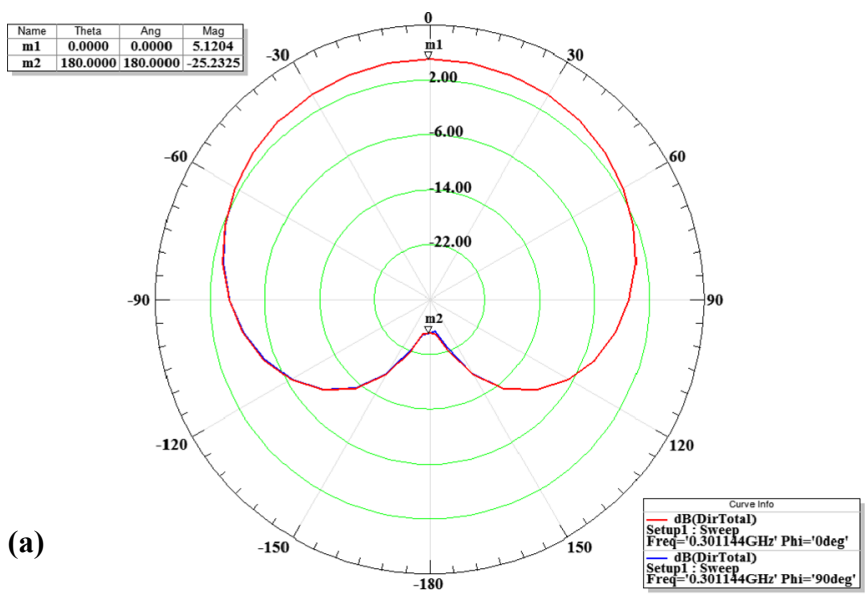

(b)
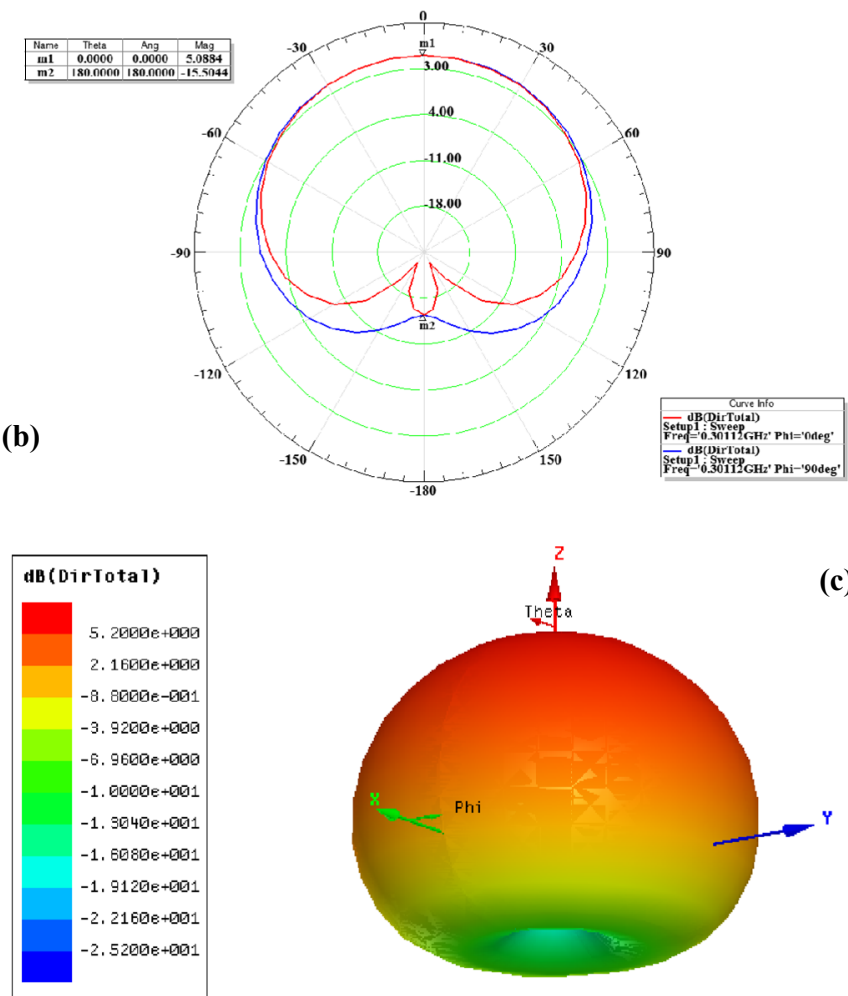

Fig. 22. The directivity patterns of the low profile NFRP Huygens source antenna. 2D E- and H-plane patterns at (a) $301.145 \mathrm{MHz}$ and (b) $301.120 \mathrm{MHz}$, and (c) 3D directivity pattern at $301.145 \mathrm{MHz}$.

\section{Conclusion}

As the demand for electrically smaller antenna systems increases, one must not forget directivity as one possible enhanced performance characteristic. The presented comparisons of several antennas, both standard and metamaterial-inspired, have given further insights into directivity as an important figure of merit, even for electrically small antennas. Advantages of combinations of driven and NFRP elements to enhance directivity were emphasized. High directivity from broadside radiating, low profile Huygens source designs, both as compact arrays and 
individual systems, was demonstrated. Of course, fabrication and measurement facility issues would have to be considered for their actual realization. Nonetheless, their predicted behaviors are interesting and are potentially useful. The ability to have electrically small antennas that radiate efficiently in the broadside direction with no back radiation opens exciting new avenues for immediate applications in a variety of commercial and military wireless systems.

\section{References}

1. S.R. Best, A discussion on small antennas operating with small finite ground planes, in: Proc. 2006 IEEE International Workshop on Antenna Technology: Small Antennas and Novel Metamaterials, iWAT2006, White Plains, NY, 2006, pp. $152-155$

2. P.-S. Kildal, Fundamental directivity and efficiency limitations of single- and multi-port antennas, in: Proc. the Second European Conference on Antennas and Propagation, EuCAP 2007, IET Conference Publications, Edinburgh, UK, 2007, pp. 1-6

3. P.-S. Kildal, S.R. Best, Further investigations of fundamental directivity limitations of small antennas with and without ground planes, in: Proc. 2008 IEEE Antennas and Propagation Society International Symposium, IEEE, San Diego, CA, 2008, pp. 1-4.

4. S.R. Best, D.L. Hanna, Design of a broadband dipole in close proximity to an EBG ground plane, IEEE Antennas Propag. Mag. 50(6), 52-64 (2008)

5. A.D. Yaghjian, T.H. O'Donnell, E.E. Altshuler, S.R. Best, Electrically small supergain end-fire arrays, Radio Sci. 43 (RS3002), 1-13 (2008)

6. S.R. Best, E.E. Altshuler, A.D. Yaghjian, J.M. McGinthy, T. H. O'Donnell, An impedance-matched 2-element superdirective array, IEEE Antennas Wirel. Propag. Lett. 7, 302-305 (2008)

7. 7. S. Lim, H. Ling, Design of electrically small Yagi antenna, Electron. Lett. 43(5), 3-4 (2007)

8. S. Lim, H. Ling, Design of a closely spaced, folded Yagi antenna, IEEE Antennas Wirel. Propag. Lett. 5, 302-305 (2006)

9. F. Yang, Y. Rahmat-Samii, Electromagnetic band gap structures in antenna engineering (Cambridge University Press, Cambridge, UK, 2009)

10. R.F. Jimenez Broas, D.F. Sievenpiper, E. Yablonovitch, A high-impedance ground plane applied to a cellphone handset geometry, IEEE Trans. Microwave Theory Tech. 49(7), 1262-1265(2001)

11. S. Clavijo, R.E. Diaz, W.E. McKinzie, Design methodology for Sievenpiper high-impedance surfaces: an artificial magnetic conductor for positive gain electrically small antennas, IEEE Trans. Antennas Propag. 51(10), 2678-2690 (2003)

12. R. Coccioli, F.-R. Yang, K.-P. Ma, T. Itoh, Aperture-coupled patch antenna on UC-PBG substrate, IEEE Trans. Microwave Theory Tech. 47(11), 2123-2130 (1999)

13. A. Erentok, P. Luljak, R.W. Ziolkowski, Antenna performance near a volumetric metamaterial realization of an artificial magnetic conductor, IEEE Trans. Antennas Propag. 53(1), 160-172 (2005)

14. R.W. Ziolkowski, P. Jin, C.-C. Lin, Metamaterial-inspired engineering of antennas, Proc. IEEE 99(10), 1720-1731 (2011)
15. M.-C. Tang, R.W. Ziolkowski, Efficient, high directivity, large front-to-back-ratio, electrically small, near-field-resonant-parasitic antenna, IEEE Access 1(1), 16-28 (2013)

16. M.-C. Tang, R.W. Ziolkowski, S. Xiao, M. Li, A highdirectivity, wideband, efficient, electrically small antenna system, IEEE Trans. Antennas Propag. 62(12), 6541-6547 (2014)

17. R.W. Ziolkowski, M.-C. Tang, N. Zhu, An efficient, broad bandwidth, high directivity, electrically small antenna, Microw. Opt. Technol. Lett. 55(6), 1430-1434 (2013)

18. M.-C. Tang, R.W. Ziolkowski, A compact, two-element array with ultra-high broadside directivity, IET Microw. Antennas Propag. 7(8), 663-671 (2013)

19. M.-C. Tang, R.W. Ziolkowski, Two-element Egyptian axe dipole arrays emphasising their wideband and end-fire radiation performance, IET Microw. Antennas Propag. 9 (13), 1363-1370 (2015)

20. P. Jin, R.W. Ziolkowski, Metamaterial-inspired, electrically small Huygens sources, IEEE Antennas Wirel. Propag. Lett. 9, 501-505 (2010)

21. T. Niemi, P. Alitalo, A.O. Karilainen, S.A. Tretyakov, Electrically small Huygens source antenna for linear polarization, IET Microw. Antennas Propag. 6(7), 735-739 (2012)

22. R.W. Ziolkowski, Low profile, broadside radiating, electrically small Huygens source antennas, IEEE Access 3, 26442651 (2015)

23. M.-C. Tang, H. Wang, R.W. Ziolkowski, Design and testing of simple, electrically small, low-profile, Huygens source antennas with broadside radiation performance, IEEE Trans. Antennas Propag. 64(11), 4607-4617 (2016)

24. M.-C. Tang, T. Shi, R.W. Ziolkowski, Electrically small, broadside radiating Huygens source antenna augmented with internal non-Foster elements to increase its bandwidth, IEEE Antennas Wirel. Propag. Lett. 16, 712-715 (2016)

25. M.-C. Tang, B. Zhou, R.W. Ziolkowski, Low-profile, electrically small, Huygens source antenna with patternreconfigurability that covers the entire azimuthal plane, IEEE Trans. Antennas Propag. 65(3), 1063-1072 (2017)

26. ANSYS High Frequency Structure Simulator (HFSS), http://www.ansys.com/Products/Electronics/ANSYSHFSS, 2016

27. G.A. Deschamps, Microstrip microwave antennas, in: Proc. Third Symposium on the USAF Antenna Research and Development Program, Robert Allerton Park, IL, 1953

28. Y.T. Lo, D. Soloman, W.F. Richards, Theory and experiment on microstrip antennas, in: Proc. 1978 Antenna Applications Symposium, Robert Allerton Park, IL, 1978

29. Y.T. Lo, D. Soloman, W.F. Richards, Theory and experiment on microstrip antennas, IEEE Trans. Antennas Propag. AP27(3), 137-145 (1979)

30. W.F. Richards, Y.T. Lo, D. Harrison, An improved theory for microstrip antennas and applications, IEEE Trans. Antennas Propag. AP-29(1), 38-46 (1981)

31. C.A. Balanis, Antenna theory, 3rd ed. (John Wiley \& Sons, Hoboken, NJ, 2005)

32. A. Erentok, R.W. Ziolkowski, Metamaterial-inspired efficient electrically-small antennas, IEEE Trans. Antennas Propag. 56(3), 691-707 (2008)

33. A.K. Bhattacharyya, Effects of finite ground plane on the radiation characteristics of a circular patch antenna, IEEE Trans. Antennas Propag. 38(2), 152-159 (1990) 
34. S.I. Latif, L. Shafai, Pattern equalization of circular patch antennas using different substrate permittivities and ground plane sizes, IEEE Trans. Antennas Propag. 59(10), 35023511 (2011)

35. D. Sievenpiper, L. Zhang, R.F. Broas, N.G. Alexopolous, E. Yablonovitch, High-impedance electromagnetic surfaces with a forbidden frequency band, IEEE Microw. Theory Techn. 47(11), 2059-2074 (1999)

36. R. Gonzalo, P. De Maagt, M. Sorolla, Enhanced patchantenna performance by suppressing surface waves using photonic-bandgap substrates, IEEE Microw. Theory Techn. 47(11), 2131-2138 (1999)

37. F. Yang, Y. Rahmat-Samii, Electromagnetic band gap structures in antenna engineering (Cambridge University Press, Cambridge, UK, 2009)

38. M.G. Silveirinha, C.A. Fernandes, J.R. Costa, Electromagnetic characterization of textured surfaces formed by metallic pins, IEEE Trans. Antennas Propag. 56(2), 405-411 (2008)

39. S. Sun, Q. He, S. Xiao, Q. Xu, X. Li, L. Zhou, Gradient-index meta-surfaces as a bridge linking propagating waves and surface waves, Nat. Mater. 11(5), 426-431 (2012)

40. A.V. Kildishev, A. Boltasseva, V.M. Shalaev, Planar photonics with metasurfaces, Science 339 (6125), 1232009 (2013)

41. L. La Spada, T.M. McManus, A. Dyke, S. Haq, L. Zhang, Q. Cheng, Y. Hao, Surface wave cloak from graded refractive index nanocomposites, Sci. Rep. 6, 29363 (2016)

42. R.W. Ziolkowski, C.-C. Lin, J.A. Nielsen, M.H. Tanielian, C. L. Holloway, Design and experimental verification of a $3 \mathrm{D}$ magnetic EZ antenna at $300 \mathrm{MHz}$, IEEE Antennas Wirel. Propag. Lett. 8, 989-993 (2009)

43. C.-C. Lin, R.W. Ziolkowski, J.A. Nielsen, M.H. Tanielian, C. L. Holloway, An efficient, low profile, electrically small, three-dimensional, very high frequency magnetic EZ antenna, Appl. Phys. Lett. 96(10), 104102 (2010)

44. J. Church, J.-C.S. Chieh, L. Xu, J.D. Rockway, D. Arceo, UHF electrically small box cage loop antenna with an embedded non-Foster load, IEEE Antennas Wirel. Propag. Lett. 13, 1329-1332 (2014)

45. R.W. Ziolkowski, Propagation in and scattering from a matched metamaterial having a zero index of refraction, Phys. Rev. E 70, 046608 (2004)

46. I. Liberal, N. Engheta, Nero-zero refractive index photonics, Nat. Photon. 11(3), 149-158 (2017)

47. T.M. McManus, L. La Spada, Y. Hao, Isotropic and anisotropic surface wave cloaking techniques, J. Opt. 18 (4), 044005 (2016)

48. R.T. Cutshall, R.W. Ziolkowski, Performance characteristics of planar and three-dimensional versions of a frequency-agile electrically small antenna, IEEE Antennas Propag. Mag. 56 (6), 53-71 (2014)

49. C.C. Lin, R.W. Ziolkowski, J.A. Nielsen, M.H. Tanielian, C. L. Holloway, An efficient, low profile, electrically small, three-dimensional, very high frequency magnetic EZ antenna, Appl. Phys. Lett. 96(10), 104102 (2010)

50. Z. Li, Z. Du, M. Takahashi, K. Saito, K. Ito, Reducing mutual coupling of MIMO antennas with parasitic elements for mobile terminals, IEEE Trans. Antennas Propag. 60(2), 473-481 (2012)

51. B.K. Lau, J.B. Andersen, Simple and efficient decoupling of compact arrays with parasitic scatterers, IEEE Trans. Antennas Propag. 60(2), 464-472 (2012)
52. M.-C. Tang, S. Xiao, T. Deng, B.-Z. Wang, Parasitic patch of the same dimensions enabled excellent performance of microstrip antenna array, Appl. Comput. Electromagn. Soc. J. 25(10), 862-866 (2010)

53. F. Yang, Y. Rahmat-Samii, Microstrip antennas integrated with electromagnetic band-gap (EBG) structures: a low mutual coupling design for array applications, IEEE Trans. Antennas Propag. 51(10), 2936-2946 (2003)

54. G. Expósito-Domínguez, J.-M. Fernández-Gonzalez, P. Padilla, M. Sierra-Castañer, Mutual coupling reduction using EBG in steering antennas, IEEE Antennas Wirel. Propag. Lett. 11, 1265-1268 (2012)

55. S. Xiao, M.-C. Tang, Y.-Y. Bai, S. Gao, B.-Z. Wang, Mutual coupling suppression in microstrip array using defected ground structure, IET Microw. Antennas Propag. 5(2), 1488-1494 (2011)

56. M.-C. Tang, S. Xiao, T. Deng, B.-Z. Wang, Novel folded single split ring resonator and its application to eliminate scan blindness in infinite phased array, in: Proc. 2010 International Symposium on Signals, Systems and Electronics (ISSSE2010), Nanjing, PR China, 2010, pp. 1-4

57. M.-C. Tang, S. Xiao, B.-Z. Wang, J. Guan, T. Deng, Improved performance of a microstrip phased array using broadband and ultra-low-loss metamaterial slabs, IEEE Antennas Propag. Mag. 53(6), 31-41 (2011)

58. D.B.M. Trindade, C. Müller, M.C.F.D. Castro, F.C.C.D Castro, Metamaterials applied to ESPAR antenna for mutual coupling reduction, IEEE Antennas Wirel. Propag. Lett. 14, 430-433 (2015)

59. Z. Qamar, U. Naeem, S.A. Khan, M. Chongcheawchamnan, M.F. Shafique, Mutual coupling reduction for high-performance densely packed patch antenna arrays on finite substrate, IEEE Trans. Antennas Propag. 64(5), 16531660 (2016)

60. R. Hafezifard, M. Naser-Moghadasi, J.R. Mohassel, R.A. Sadeghzadeh, Mutual coupling reduction for two closely spaced meander line antennas using metamaterial substrate, IEEE Antennas Wirel. Propag. Lett. 15, 40-43 (2016)

61. B. Wu, H. Chen, J.A. Kong, T.M. Grzegorczyk, Surface wave suppression in antenna systems using magnetic metamaterial, J. Appl. Phys. 101, 112913(1)-112913(4) (2007)

62. X.M. Yang, X.G. Liu, X.Y. Zhou, T.J. Cui, Reduction of mutual coupling between closely packed patch antennas using waveguided metamaterials, IEEE Antennas Wirel. Propag. Lett. 11, 389-391 (2012)

63. P.J. Ferrer, J.M. González-Arbesú, J. Romeu, Decorrelation of two closely spaced antennas with a metamaterial AMC surface, Microw. Opt. Technol. Lett. 50(5), 1414-1417 (2008)

64. M. Imbert, P.J. Ferrer, J.M. González-Arbesú, J. Romeu, Assessment of the performance of a metamaterial spacer in a closely spaced multiple-antenna system, IEEE Antennas Wirel. Propag. Lett. 11, 720-723 (2012)

65. G. Zhai, Z.N. Chen, X. Qing, Enhanced isolation of a closely spaced four-element MIMO antenna system using metamaterial mushroom, IEEE Trans. Antennas Propag. 63(8), 3362-3370 (2015)

66. M.C. Tang, Z. Chen, H. Wang, M. Li, B. Luo, J. Wang, Z. Shi, R.W. Ziolkowski, Mutual coupling reduction using metastructures for wideband, dual-polarized, high-density patch arrays, IEEE Trans. Antennas Propag. 65(8), 3986-3998 (2017) 
67. A. Alù, N. Engheta, Polarizabilities and effective parameters for collections of spherical nanoparticles formed by pairs of concentric double-negative, single-negative, and/or doublepositive metamaterial layers, J. Appl. Phys. 97(9), 094310 (2015)

68. J.A. Gordon R.W. Ziolkowski, CNP optical metamaterials, Opt. Express 16(9), 6692-6716 (2008)

69. C.R. Simovski, S.A. Tretyakov, Model of isotropic resonant magnetism in the visible range based on core-shell clusters, Phys. Rev. B 79(4), 045111 (2009)

70. R. Paniagua-Domínguez, F. López-Tejeira, R. Marqués, J.A. Sánchez-Gil, Metallo-dielectric core-shell nanospheres as building blocks for optical three-dimensional isotropic negative-index metamaterials, New J. Phys. 13(12), 123017 (2011)

71. R. Tarparelli, R. Iovine, L. La Spada, L. Vegni, Surface plasmon resonance of nanoshell particles with PMMAgraphene core, COMPEL 33(6), 2016-2029 (2014)

72. F. Monticone, A. Alù, Metamaterial, plasmonic and nanophotonic devices, Rep. Prog. Phys. 80(3), 036401 (2017)

73. R.W. Ziolkowski, A. Erentok, At and below the Chu limit: passive and active broad bandwidth metamaterial-based electrically small antennas, IET Microw. Antennas Propag. 1 (1), 16-128 (2007)

Cite this article as: Richard W. Ziolkowski, The directivity of a compact antenna: an unforgettable figure of merit, EPJ Appl. Metamat. 2017, 4, 7 